\title{
Conocimiento acerca de las estrategias de práctica instrumental al inicio del Grado Superior de Música
}

\author{
Knowledge about the instrumental practice strategies at the beginning of the Bachelor \\ of Music Degree
}

\section{Resumen}

Cabe pensar que un estudiante de instrumento que accede al Grado Superior de Música, tras aproximadamente 10 años de formación, debe tener una práctica efectiva y, por tanto, un manejo adecuado de numerosas estrategias. En este sentido, el estudio se planteó con objeto de conocer el estado de conocimientos previos de los estudiantes que ingresan al Conservatorio Superior de Música de Aragón con respecto a diversas estrategias de práctica instrumental (p. ej., práctica mental, alta concentración, escucha autocrítica, práctica segmentada...) de eficacia avalada por investigaciones previas y/o la experiencia profesional de grandes intérpretes. El estudio de carácter descriptivo presenta una metodología de carácter cualitativo. Se llevó a cabo un cuestionario y una entrevista personal a 28 estudiantes con una posterior triangulación de los resultados obtenidos. Los resultados evidencian que, de media, tan sólo el $38.89 \%$ de los estudiantes conocían y manejaban las estrategias de práctica instrumental propuestas antes de su participación. De modo que, los contenidos del programa didáctico implementado eran, en general, desconocidos, sobre todo para los instrumentistas de viento-metal. En resumen, los hallazgos obtenidos alertan acerca de la necesidad de formar a los estudiantes de Grado Superior en la práctica estratégica con objeto de optimizar su estudio individual.

Palabras clave: Estrategias de Práctica Instrumental, Estudio Individual, Instrumento, Formación Superior

\begin{abstract}
One would imagine that an instrumental student who embarks on the pursuit of a Bachelor of Music degree, after approximately 10 years of education, should have an effective practice routine and, therefore, an adequate management of numerous strategies. Along these lines, this study was designed, in order to corroborate the state of previous knowledge of new students of the Music Conservatory of Aragon with respect to various strategies of instrumental practice (e.g., mental practice, high concentration, selfcritical audiation, and segmented practice) of effectiveness endorsed by previous research and / or professional experience of Great interpreters. The descriptive study presents a qualitative methodology. Twenty-eight students have done the questionnaire and the personal interview, all followed by a triangulation of data. The results show that, on average, only $38.89 \%$ of the students knew and managed the proposed practice strategies before their participation. Thus, the contents of the implemented didactic program were, generally unknown, especially among brass players. In summary, the findings obtained warn of the need to train postsecondary students in the strategic practice capabilities, in order to optimize their individual study.
\end{abstract}

Key words: Instrumental Practice Strategies, Individual Practice, Instrument, Higher Education.

THE CONTENT OF THIS ARTICLE IS THE SOLE RESPONSIBILITY OF THE AUTHORS. THE REVISTA ELECTRÓNICA DE LEEME AND UNIVERSITAT DE VALĖNCIA ARE NOT LIABLE FOR ANY LEGAL ACTIONS THAT MAY ARISE INVOLVING THE ARTICLE'S CONTENT. REVISTA ELECTRÓNICA DE LEEME -LISTA ELECTRÓNICA EUROPEA DE MÚSICA EN LA EDUCACIÓNHTTP://MUSICA.REDIRIS.ES.ISSN: 1575-9563 EDITORES: UNIVERSIDAD DE VALENCIA Y JESÚS TEJADA GIMÉNEZ. VISIBILIDAD DE ESTA REVISTA: EBSCO, CINDOC (CESIC), CITEFACTOR, COPAC, DIALNET, DICE (CSIC), DOAJ, E-REVISTAS (CSIC), EBSCO PREMIER, ERIH+, GALE CENGAGE LEARNING, IN-RECS, IRESIE, LATINDEX, MIAR, OCLC WORLDCAT, RESH, REDIB, RILM CORE JOURNALS, SUDOC, ULRICHS, ZEITSCHRIFTDATENBANK, ESTA REVISTA ESTÁ PUBLICADA CON EL APOYO INSTITUCIONAL DE REDIRIS-CONSEJO SUPERIOR DE INVESTIGACIONES CIENTIFICAS Y ES DE ACCESO LIBRE. 


\section{Introducción}

Uno de los retos más apasionantes para los docentes e investigadores musicales consiste en hallar estrategias de ayuda para que los intérpretes puedan satisfacer sus demandas físicas y mentales de modo eficiente y eficaz. En definitiva, buscar estrategias de práctica instrumental entendidas como:

[...] aquellos pensamientos y comportamientos que, de forma consciente e intencional, guían al intérprete durante la práctica deliberada de su instrumento e intervienen en el modo en el que selecciona, organiza, procesa, integra y ejecuta sus conocimientos y habilidades musicales; en su estado emocional; y/o en su motivación; con el propósito de adquirir, almacenar y posteriormente poder reproducir resultados instrumentales, a su juicio, positivos y en el menor tiempo de consecución posible. (Tripiana, 2012:66)

Reid (2006) manifiesta que, a pesar de la naturaleza subjetiva de la interpretación, existen recursos específicos o estrategias gracias a las cuales los instrumentistas pueden desarrollar sus habilidades y mejorar su práctica. Para llegar a esta conclusión, observa que una característica que comparten los métodos de los intérpretes más experimentados es el enfoque sistemático del aprendizaje musical. Dicho enfoque implica una conciencia firme hacia los objetivos de la práctica instrumental; así como, la habilidad de manejar estrategias eficaces que permitan lograr los fines musicales previamente planteados.

El presente proyecto tuvo su origen en diversos interrogantes que fueron surgiendo en la práctica docente. De modo que, las cuestiones de investigación son:

- Los estudiantes de instrumento que ingresan en el Grado Superior de Música, ¿conocen las estrategias de práctica instrumental de acreditada eficacia usadas por los intérpretes profesionales?

- ¿ ¿Utilizan dichas estrategias durante su práctica instrumental habitual?

- ¿Existen diferencias en cuanto al conocimiento de las mismas en función de la especialidad instrumental?

En definitiva, el intento de dar respuesta a las cuestiones planteadas no es más que el punto de partida para dar visibilidad a la existencia o no de unos conocimientos sólidamente fundamentados, que orienten al instrumentista durante su práctica y le faciliten la experiencia de progreso en la obtención de resultados musicales óptimos. 


\section{Revisión teórica}

Por varias razones metodológicas y tecnológicas, el progreso con respecto a las estrategias de práctica instrumental ha sido, según Dunsby (1995), muy lento en comparación con otras disciplinas. De todos modos, según Williamon (2004), cada vez son más los investigadores que están forjando colaboraciones interdisciplinarias y generando métodos innovadores para la investigación de cómo la interpretación musical excepcional se puede llevar a cabo.

Con respecto a la práctica instrumental, Sloboda, Davidson, Howe y Moore (1996) evidencian que, así como el tiempo de estudio varía en las diferentes etapas de la vida de los músicos, estos tienden a desarrollar estrategias útiles relativamente tarde. Harnischmacher (1997) llega todavía más lejos al describir cuatro fases de desarrollo de las mismas:

- $\quad$ Durante la primera etapa o fase de actividad (8 a 10 años), señala que la práctica se distingue por el componente lúdico como una forma autogenerada de acción.

- $\quad$ En la etapa de adopción (11 a 12 años), el sujeto adopta una ética de trabajo impuesta desde el exterior y comienza a reflexionar sobre la causalidad y la orientación hacia una meta de la práctica instrumental.

- Durante la etapa de asimilación (13 a 14 años), el estudio musical se convierte en una tarea integrada en su programación diaria.

- $\quad$ En la fase de identificación (15 a 18 años), el estudiante considera la propia orientación de su práctica y el aumento de la calidad y la economía de la misma adoptan un papel más relevante.

En 1988, Gruson observó y analizó el comportamiento de los estudiantes de Piano durante la práctica por medio de una entrevista con la que pretendía evidenciar el conocimiento sobre la misma en función de su experiencia musical. Según sus resultados, los músicos más experimentados fueron capaces de conceptualizar su comportamiento en el estudio de una manera más diferenciada y abstracta. Se describieron más estrategias de práctica instrumental y resultaron ser más complejas cognitivamente.

Otro estudio exploratorio (Byo y Cassidy, 2008), recogió información acerca de estudiantes de diversas especialidades instrumentales y reveló gran variedad de estrategias que decían emplear durante su estudio: tocar lento, efectuar cambios, aislar dificultades, usar metrónomo, efectuar comentarios generales, repetir fragmentos, analizar música, memorizar, grabarse, estudiar escalas... Los participantes practicaron durante 50 minutos y, lo realmente interesante fue que, al visualizar las grabaciones aparecieron estrategias que no habían sido detalladas durante la recopilación de datos previa como silbar, cantar, tocar solo con la

THE CONTENT OF THIS ARTICLE IS THE SOLE RESPONSIBILITY OF THE AUTHORS. THE REVISTA ELECTRÓNICA DE LEEME AND UNIVERSITAT DE VALĖNCIA ARE NOT LIABLE FOR ANY LEGAL ACTIONS THAT MAY ARISE INVOLVING THE ARTICLE'S CONTENT. REVISTA ELECTRÓNICA DE LEEME -LISTA ELECTRÓNICA EUROPEA DE MÚSICA EN LA EDUCACIÓNHTTP://MUSICA.REDIRIS.ES.ISSN: 1575-9563 EDITORES: UNIVERSIDAD DE VALENCIA Y JESÚS TEJADA GIMÉNEZ. VISIBILIDAD DE ESTA REVISTA: EBSCO, CINDOC (CESIC), CITEFACTOR, COPAC, DIALNET, DICE (CSIC), DOAJ, E-REVISTAS (CSIC), EBSCO PREMIER, ERIH+, GALE CENGAGE LEARNING, IN-RECS, IRESIE, LATINDEX, MIAR, OCLC WORLDCAT, RESH, REDIB, RILM CORE JOURNALS, SUDOC, ULRICHS, ZEITSCHRIFTDATENBANK, ESTA REVISTA ESTÁ PUBLICADA CON EL APOYO INSTITUCIONAL DE REDIRIS-CONSEJO SUPERIOR DE INVESTIGACIONES CIENTIFICAS Y ES DE ACCESO LIBRE. 
embocadura, añadir palabras a la melodía o al ritmo, mover los dedos, usar un espejo... De modo que, mientras los participantes explicaban en la entrevista estrategias muy conocidas en el ámbito instrumental, ellos realmente usaban otras muy diferentes en su práctica habitual o al menos eso fue lo que se observó en las grabaciones.

Varios estudios declaran que los intérpretes de música poseen un conocimiento limitado de estrategias específicas de práctica y, al mismo tiempo, evidencian la existencia de diferencias considerables entre principiantes, instrumentistas novatos e instrumentistas expertos (Hallam, 2001; Pitts, Davidson, y McPherson, 2002; Renwick y McPherson, 2002). Según Jørgensen (2004), el desarrollo de un repertorio de estrategias de práctica y la adquisición de conocimientos acerca de las mismas y de sus funciones cognitivas propias es uno de los objetivos más importantes para cualquier instrumentista interesado en el ejercicio docente.

Finalmente, tras la oportuna revisión teórica, se presentan los objetivos que persigue el presente estudio. Son los siguientes:

- $\quad$ Evidenciar si los participantes poseían un conocimiento previo acerca de cada una de las 21 estrategias de práctica instrumental de acreditada eficacia que se presentan en el estudio.

- Describir la existencia de diferencias, con respecto al objetivo anterior, en función de la familia instrumental analizada.

\section{Método}

El objeto del presente trabajo era evidenciar los conocimientos previos de los estudiantes de Grado Superior con respecto a 21 estrategias de práctica instrumental de eficacia avalada por grandes figuras de la docencia, testimonios de grandes intérpretes y/o recientes estudios. Para ello, la investigación de carácter descriptivo presenta un diseño de carácter cualitativo en la que las técnicas de recogida de datos han sido el cuestionario y la entrevista personal.

\subsection{Muestra}

En la Tabla 1, se presentan los agentes implicados en la investigación. Son 28 estudiantes del Conservatorio Superior de Música de Aragón seleccionados mediante un muestreo aleatorio estratificado para verificar que todas las franjas de interés, es decir, que las diversas especialidades instrumentales estuvieran representadas adecuadamente en la muestra. 
Tabla 1. Distribución de instrumentistas participantes

\begin{tabular}{ccc}
\hline & Hombres & Mujeres \\
\hline Primer curso & 11 & 4 \\
Segundo curso & 6 & 7 \\
\hline Total & 17 & 11 \\
\hline
\end{tabular}

Los estudiantes fueron invitados a participar en esta investigación y mostraron su conformidad por medio de la firma de un consentimiento informado en el que quedaba reflejada la justificación, objetivos, beneficios, procedimientos, dificultades asociadas y todas las aclaraciones pertinentes acerca del estudio.

\subsection{Diseño}

La investigación se desarrolló en varias fases. En primer lugar, se efectuó un exhaustivo análisis documental para hallar estrategias de práctica instrumental de eficacia avalada. En segundo lugar, se elaboró un programa didáctico acerca de las mismas. Por último, se implementó dicho programa con 28 estudiantes de Grado Superior.

\subsubsection{Búsqueda de estrategias de práctica instrumental}

En la primera de ellas, se establecieron las bases para elaborar un programa didáctico sobre estrategias de práctica instrumental. El propósito era hallar propuestas avaladas en su eficacia por grandes figuras de la docencia, el testimonio de grandes intérpretes y recientes investigaciones. Después de una exhaustiva revisión documental, se identificaron 21 estrategias de práctica instrumental que, tras su conveniente diseño, se constituyeron en el eje principal del programa didáctico. A continuación, en la Tabla 2, se describe brevemente cada una de ellas, así como, los autores que avalan su eficacia:

Tabla 2. Estrategias de práctica instrumental identificadas

\begin{tabular}{|c|c|}
\hline Estrategia de práctica & Autores \\
\hline $\begin{array}{l}\text { 1-Práctica fragmentada. Conjunto de pensamientos o } \\
\text { pautas de conducta planificados sistemáticamente, que } \\
\text { son llevados a cabo por el instrumentista de forma } \\
\text { intencional para lograr un resultado musical satisfactorio } \\
\text { por medio de la división de lo complejo y de la } \\
\text { obtención de pequeños dominios parciales previos al }\end{array}$ & $\begin{array}{l}\text { Bloomfield-Zeisler, 1913; Bunting, 1999; Burwell y } \\
\text { Shipton, 2013; Calvo-Manzano, 1987; Capistrán, 2017; } \\
\text { Carlevaro, 2000; Casas, 2013; Chafin y Imreh, 2001; } \\
\text { Chaffin y Lemieux, 2004; Chaffin, Lisboa, Logan y } \\
\text { Begosh, 2010; Coso,1992; Coyle, 2009; Gabrielsson, } \\
\text { 2003; Galamian, 1998; Ginsborg, 2004; Gruson, 1988; }\end{array}$ \\
\hline
\end{tabular}

THE CONTENT OF THIS ARTICLE IS THE SOLE RESPONSIBILITY OF THE AUTHORS. THE REVISTA ELECTRÓNICA DE LEEME AND UNIVERSITAT DE VALĖNCIA ARE NOT LIABLE FOR ANY LEGAL ACTIONS THAT MAY ARISE INVOLVING THE ARTICLE'S CONTENT. REVISTA ELECTRÓNICA DE LEEME -LISTA ELECTRÓNICA EUROPEA DE MÚSICA EN LA EDUCACIÓNHTTP://MUSICA.REDIRIS.ES.ISSN: 1575-9563 EDITORES: UNIVERSIDAD DE VALENCIA Y JESÚS TEJADA GIMÉNEZ. VISIBILIDAD DE ESTA REVISTA: EBSCO, CINDOC (CESIC), CITEFACTOR, COPAC, DIALNET, DICE (CSIC), DOAJ, E-REVISTAS (CSIC), EBSCO PREMIER, ERIH+, GALE CENGAGE LEARNING, IN-RECS, IRESIE, LATINDEX, MIAR, OCLC WORLDCAT, RESH, REDIB, RILM CORE JOURNALS, SUDOC, ULRICHS, ZEITSCHRIFTDATENBANK, ESTA REVISTA ESTÁ PUBLICADA CON EL APOYO INSTITUCIONAL DE REDIRIS-CONSEJO SUPERIOR DE INVESTIGACIONES CIENTIFICAS Y ES DE ACCESO LIBRE. 
dominio completo, dentro de un plan de retroalimentación tras la oportuna concepción, puesta en práctica y evaluación.

2-Practicar con máxima concentración durante la ejecución instrumental, es decir, manteniendo una conciencia constante sobre un tema específico excluyendo momentáneamente otras cuestiones.

3-Adecuación ergonómica, funcionamiento cómodo y natural del cuerpo del instrumentista por medio del movimiento natural, la economía motriz con máximo rendimiento y mínimo esfuerzo, la influencia de la gravedad, integrando la respiración y buscando el apoyo para obtener seguridad.

4-Manejo de mensajes constructivos de auto-orientación que plantean objetivos durante el estudio, sirven de auto-guía por medio de mensajes de advertencia, de apoyo o de recuerdo y efectúan una retroalimentación informativa evaluando los resultados o la sesión de

5-Escucha autocrítica, es decir, percepción auditiva atenta y objetiva, dentro de un plan de retroalimentación tras la oportuna concepción, puesta en práctica y evaluación. El instrumentista ha de percibir cómo cada sonido resuena en el espacio exterior, efectuando una adecuada autocrítica y siendo ayudado por grabaciones

6-Lectura precisa tocando con corrección desde la primera vez, aislando la dificultad, analizando su naturaleza y asumiendo un margen de aceptabilidad sin obsesionarse con la perfección. práctica. audiovisuales.

Hallam, 2004; Hinsom, 1995; Jørgensen, 2004; Miklaszewski, 1989; Miller, 1956; Mishra, 2005; Neuhaus, 1987; Reid, 2006; Rosenbaum, Kenny y Derr, 1983; Williamon y Valentine; 2000, 2002.

Ballesteros, 2004; Barrie, 2007; Bauer, 1913; Burwell y Shipton, 2013; Calvo-Manzano, 1987; Carlevaro, 2000; Chaffin y Lemieux, 2004; Connolly y Williamon, 2004); Coso,1992; Csikszentmihalyi, 1990; Derbez, 2015; Foldes, 1992; Galamian, 1998; García-Rico, 2009; Gordon, 2003; Hoppenot, 2000; Klöppel, 2005; Michalak, 2006; Neuhaus, 1987; Pascuali y Príncipe, 1982; Ramos, 1981; Reid, 2006; Sauer, 1913.

Arizcuren, 1985; Artaud, 1991; Bachmann, 1998; Ballesteros, 2004; Blum, 2000; Boehm, 1847; Bruser, 1997; Bunting, 1999; Calvo-Manzano, 1987, 2008; Carlevaro, 2000; Chenoll, 1990; Coso,1992; Dalia y Pozo, 2006; Davidson, 1993; Derbez, 2015; Fujisawa, Iwami, Kinou y Miura, 2009; Galamian, 1998; Grindea, 1991; Hoppenot, 2000; Kenny y Ackermann, 2015; Klöppel, 2005; Manresa, 2006; Martenot, 1993; Michalak, 2006; Narejos, 2000; Neuhaus, 1987; Nieto, 1999; Palmer, Carter, Koopmans y Loehr, 2007; Parncutt y Troup, 2002; Pascuali y Príncipe, 1982; Ramos, 1981; Rosset-Llobet, 2000; Ruiz, 1999; Samama-Polak, 1990; Sánchez, 2006; Stravinsky, 1977; Sauer, 1913; Todd, 1995; Vila, 2007; Vines, Krumhansl, Wanderley y Levitin, 2006; Wanderley, 2002; Willems, 1994.

Barry, 1990; Barry y Hallam, 2002; Burwell y Shipton, 2013; Capistrán, 2017; Chaffin, Imreh y Crawford, 2002; Chaffin y Lemieux, 2004; Coso,1992; Coyle, 2009; Cremaschi, 2012; Daniel, 2001; Derbez, 2015; Foldes, 1992; García-Rico, 2009; Gordon, 2003; Hallam, 1995; Hoppenot, 2000; Jørgensen, 2004; Jorquera, 2002; Liertz, 2002; Kurtz, 2007; Neuhaus, 1987; Reid, 2006; Weaver, 2005; Wilson y Roland, 2002; Wulf y Mornell, 2008.

Arizcuren, 1985; Artaud, 1991; Barrie, 2007; Barry y Hallam, 2002; Bruser, 1997; Bunting, 1999; Burwell y Shipton, 2013; Calvo-Manzano, 1987; Capistrán, 2017; Carlevaro, 2000; Casas, 2013; Chaffin y Lemieux, 2004; Coso,1992; Coyle, 2009; Cremaschi, 2012; Daniel, 2001; Eguilaz, 2009; Emond, Vinson, Singer, Barfurth y Brooks, 2006; Foldes, 1992; Galamian, 1998; Herrera y Cremades, 2014; Hoppenot, 2000; Manresa, 2006; Neuhaus, 1987; Pascuali y Príncipe, 1982; Pertzborn, Coimbra, Hallam y Braga, 2009; Pfordresher, 2006; Pozo, Bautista y Torrado, 2008.

Arizcuren, 1985; Bloomfield-Zeisler, 1913; Blum, 2000; Boehm, 1847; Brodsky, 2006; Bruser, 1997; Bunting, 1999; Colomer, 1990; Coso,1992; Coyle, 2009; Dalia y Pozo, 2006; Dobson, 2010;, 1992; Galamian, 1998; García-Rico, 2009; Goebl y Palmer, 2008; Gordon, 2003; Gruson, 1988; Hallam, 1995; Jørgensen, 2004; Martenot, 1993; Michalak, 2006; Neuhaus, 1987; Palmer y Drake, 1997; Pascuali y Príncipe, 1982; Ramos, 1981; Reid, 2006; Ruiz, Jabusch y Altenmüller, 2009; Stravinsky, 1977.

7-Práctica indirecta alterando el tempo de la Altenmüller y Gruhn, 2002; Arizcuren, 1985; Ballesteros, interpretación, generando nuevos problemas por 2004; Barrie, 2007; Barry y McArthur, 1994; Bengtsson, 
resolver (propuestas de ejercicios técnicos acerca del fragmento musical a trabajar) y acrecentando la dificultad.

8-Adaptación al límite técnico-interpretativo actual, es decir, no pretender más de lo que en el momento presente el instrumentista es capaz de hacer, adaptándose a su techo técnico-interpretativo.

9-Práctica del detalle que consiste en trabajar en profundidad la calidad del sonido puntual (calidad en cada nota o acorde), prestar máxima atención a los detalles que tienen lugar a lo largo de una frase musical (calidad en motivos musicales). Y, por último, trabajar minuciosamente el conjunto de la obra o sección completa esperando la crítica (calidad a gran escala).

10-Práctica técnica, es decir, buscar el equilibrio entre la práctica constructiva y la práctica interpretativa, así como manejar la técnica al servicio de la música.

11-Análisis, comprensión e interiorización armónicoformal como ayuda al instrumentista.
Nagy, Skare, Forsman, Forssberg y Ullén; 2005; Bloomfield-Zeisler, 1913; Blum, 2000; Bruser, 1997; Burwell y Shipton, 2013; Calvo-Manzano, 1987; Capistrán, 2017; Colomer, 1990; Coyle, 2009; Eguilaz, 2009; Fields, 2006, 2008; Foldes, 1992; Galamian, 1998; Gruson, 1988; Hallam, 2004; Barry y McArthur, 1994; Hinsom, 1995; Hoppenot, 2000; Jørgensen, 2004; Mechner, 1995; Miklaszewski, 1989; Neuhaus, 1987; Palmer y Drake, 1997; Parncutt y Troup, 2002; Ramos, 1981; Reid, 2006.

Bruser, 1997; Dalia y Pozo, 2006; Foldes, 1992; Hoppenot, 2000; Kenny y Ackermann, 2015; Pachmann, 1913; Pascuali y Príncipe, 1982; Schelling, 1913; Stoeber y Eismann, 2007; Zarza-Alzugaray, Casanova-López, y Robles-Rubio, 2016)

Artaud, 1991; Blum, 2000; Boehm, 1847; Bunting, 1999; Calvo-Manzano, 1987; Foldes, 1992; Galamian, 1998; García-Rico, 2009; Gordon, 2003; Manresa, 2006; Mitchell y MacDonald, 2009; Neuhaus, 1987; Pachmann, 1913; Pascuali y Príncipe, 1982; Sánchez, 2006.

Arizcuren, 1985; Barrie, 2007; Bauer, 1913; Blum, 2000; Calvo-Manzano, 1987; Carlevaro, 2000; Chaffin, Imreh, Lemieux y Chen, 2003; Chaffin, Lisboa, Logan y Begosh, 2010; Chenoll, 1990; Derbez, 2015; Eguilaz, 2009; Foldes, 1992; Galamian, 1998; García-Rico, 2009; Hinsom, 1995; Hoppenot, 2000; Jorquera, 2002; Martenot, 1993; Narejos, 1998, 2000; Neuhaus, 1987; Pascuali y Príncipe, 1982; Pertzborn et al., 2009; Ramos, 1981; Willems, 1994.

Barry y McArthur, 1994; Bigand y Pineau, 1997; Blum,
2000; Calvo-Manzano, 1987; Capistrán, 2017; Casas, 2013; Chafin y Imreh, 1997, 2001; Chaffin, Lisboa, Logan y Begosh, 2010; Coso, 1992; Gordon, 2003; Hallam, 1995; Herrera y Cremades, 2014; Hoppenot, 2000; Lisboa, Chaffin, Schiaroli y Barrera, 2004; Macmillan, 2005; Mawer, 1999; Nieto, 1999; Nuki, 1984; Pascuali y Príncipe, 1982; Reid, 2006; Rubin-Rabson, 1941; Sánchez, 2006

12-Análisis, comprensión e interiorización de los elementos expresivos de la obra: carácter, agógica, silencios, dinámica, elementos melódicos, timbre, afinación y elementos rítmicos.

13-Apoyo al discurso musical por medio del gesto respiratorio anacrúsico previo, creando líneas musicales con un fraseo coherente y completo, acentuando correctamente y a través de la eficacia del canto.
Bachmann, 1998; Ballesteros, 2004; Blum, 2000; CalvoManzano, 1987; Carlevaro, 2000; Casas, 2013; Clarke, 1982, 1988; Colomer, 1990; Friberg y Sundberg, 1999; Goebl, Dixon, De Poli, Friberg, Bresin y Widmer, 2008; Hoppenot, 2000; Juslin, 2003; Juslin, Friberg, Schoonderwaldt, Karlsson; 2004; Manresa, 2006; Martenot, 1993; Neuhaus, 1987; Nieto, 1999; Palmer, 1989, 1997; Pascuali y Príncipe, 1982; Repp, 1992, 2001; Ruiz, 1999; Shaffer, 1981; Sloboda, , 1983, 1991; Sloboda, Minassian y Gayford, 2003; Stravinsky, 1977; Woody, 2004

Arizcuren, 1985; Ballesteros, 2004; Bauer, 1913; Besson y Schön, 2003; Blum, 2000; Boehm, 1847; Broughton, Stevens, y Malloch, 2006; Bruser, 1997; Bunting, 1999; Calvo-Manzano, 1987, 2008; Chenoll, 1990; Clarke, 1988; Colomer, 1990; Esteban, 2007; Hoppenot, 2000; MacRitchie, Buck y Bailey, 2009; Martenot, 1993; Nieto, 1999; Ohgushi, 2006; Palmer, Koopmans, Carter, Loehr y

THE CONTENT OF THIS ARTICLE IS THE SOLE RESPONSIBILITY OF THE AUTHORS. THE REVISTA ELECTRÓNICA DE LEEME AND UNIVERSITAT DE VALĖNCIA ARE NOT LIABLE FOR ANY LEGAL ACTIONS THAT MAY ARISE INVOLVING THE ARTICLE'S CONTENT. REVISTA ELECTRÓNICA DE LEEME -LISTA ELECTRÓNICA EUROPEA DE MÚSICA EN LA EDUCACIÓNHTTP://MUSICA.REDIRIS.ES.ISSN: 1575-9563 EDITORES: UNIVERSIDAD DE VALENCIA Y JESÚS TEJADA GIMÉNEZ. VISIBILIDAD DE ESTA REVISTA: EBSCO, CINDOC (CESIC), CITEFACTOR, COPAC, DIALNET, DICE (CSIC), DOAJ, E-REVISTAS (CSIC), EBSCO PREMIER, ERIH+, GALE CENGAGE LEARNING, IN-RECS, IRESIE, LATINDEX, MIAR, OCLC WORLDCAT, RESH, REDIB, RILM CORE JOURNALS, SUDOC, ULRICHS, ZEITSCHRIFTDATENBANK, ESTA REVISTA ESTÁ PUBLICADA CON EL APOYO INSTITUCIONAL DE REDIRIS-CONSEJO SUPERIOR DE INVESTIGACIONES CIENTIFICAS Y ES DE ACCESO LIBRE. 


\begin{tabular}{|c|c|}
\hline & $\begin{array}{l}\text { Wanderley, 2009; Palmer y Hutchins, 2006; } \\
\text { Papageorgiou, 2007; Parncutt, 2003; Pascuali y Príncipe, } \\
\text { 1982; Reid, 2006; Ruiz, 1999; Stravinsky, 1977; Vines, } \\
\text { Krumhansl, Wanderley y Levitin, } 2006 .\end{array}$ \\
\hline $\begin{array}{l}\text { 14-Visualización creativa, es decir, anteponer el } \\
\text { pensamiento a la acción, pensar e imaginar antes de } \\
\text { tocar. De esta estrategia, se derivan diversas propuestas; } \\
\text { se puede efectuar la visualización creativa para adoptar } \\
\text { un modelo corporal experimentado desde el interior } \\
\text { (propiocepción), para crear una imagen cinestésica } \\
\text { previa, para controlar mentalmente el movimiento } \\
\text { (dirigir y supervisar) y para experimentar la receptividad } \\
\text { sensorial interrelacionando un resultado positivo con } \\
\text { sensaciones internas. Esta práctica mental también } \\
\text { puede generar una imagen sonora previa, de modo que } \\
\text { sea posible escuchar la obra en la mente lejos del } \\
\text { instrumento. A su vez, los símbolos e imágenes } \\
\text { evocadoras, pueden propiciar la creación de un } \\
\text { programa extra-musical o la generación de una imagen } \\
\text { anticipada del tempo que también pueden ayudar al } \\
\text { intérprete. Del mismo modo, la visualización creativa } \\
\text { puede ser de utilidad para lograr una imagen escénica } \\
\text { previa, es decir, un escenario mental. }\end{array}$ & $\begin{array}{l}\text { Arizcuren, 1985; Bachmann, 1998; Ballesteros, 2004; } \\
\text { Barrie, 2007; Barry y Hallam, 2002; Bernardi, Schories, } \\
\text { Jabusch, Colombo y Altenmüller, 2009; Blum, 2000; } \\
\text { Broughton y Stevens, 2009; Bruser, 1997; Bunting, 1999; } \\
\text { Calvo-Manzano, 1987, 2008; Carlevaro, 2000; Casas, } \\
\text { 2013; Chaffin, Imreh, Lemieux y Chen, 2003; Chenoll, } \\
\text { 1990; Clark y Williamon, 2009; Clarke, 1988; Colomer, } \\
\text { 1990; Connolly y Williamon, 2004; Coso, 1992; Coyle, } \\
\text { 2009; Derbez, 2015; Driskell, Copper y Moran, 1994; } \\
\text { Foldes, 1992; Galamian, 1998; García-Rico, 2009; } \\
\text { Gordon, 2003; Haddon, 2007; Herrera y Cremades, 2014; } \\
\text { Highben y Palmer, 2004; Hinsom, 1995; Hoppenot, 2000; } \\
\text { Jørgensen, 2004; Klöppel, 2005; Liertz, 2002; Manresa, } \\
\text { 2006; Martenot, 1993; Michalak, 2006; Mishra, 2005; } \\
\text { Neuhaus, 1987; Pascual-Leone, 2003; Pascuali y } \\
\text { Príncipe, 1982; Pozo et al., 2008; Ramos, 1981; } \\
\text { Rauschecker, 2003; Repp, 2001; Reid, 2006; Ross, 1985; } \\
\text { Samama-Polak, 1990; Stravinsky, 1977; Trusheim, 1991; } \\
\text { Willems, 1994; Wilson y Roland, 2002; Woody, 2004; } \\
\text { Zatorre y Halpern, 2005. }\end{array}$ \\
\hline $\begin{array}{l}\text { 15-Fomentar la } \\
\text { medio de una eje }\end{array}$ & $\begin{array}{l}\text { Blum, 2000; Bruser, 1997; Chaffin y Lemieux, 2004; } \\
\text { Derbez, 2015; Foldes, 1992; Galamian, 1998; García- } \\
\text { Rico, 2009; Gordon, 2003; Hoppenot, 2000; Klöppel, } \\
\text { 2005; Neuhaus, 1987; Nieto, 1999; Wrigley y Emmerson, } \\
\text { 2013. }\end{array}$ \\
\hline $\begin{array}{l}\text { 16-Experimentar la transmisión de vibraciones } \\
\text { musicales del instrumento al cuerpo del instrumentista }\end{array}$ & $\begin{array}{l}\text { Bruser, 1997; Derbez, 2015; Hoppenot, 2000; Martenot, } \\
\text { 1993; Pérez Sánchez, 2003; Steiner, } 1984 .\end{array}$ \\
\hline $\begin{array}{l}\text { 17-Practicar deliberadamente la comunicación de ideas } \\
\text { musicales, es decir, tiene por objeto la transmisión del } \\
\text { contenido musical, por medio de la proyección de las } \\
\text { ideas musicales al auditorio, y dentro de un plan de } \\
\text { retroalimentación tras la oportuna concepción, puesta en } \\
\text { práctica y evaluación. }\end{array}$ & $\begin{array}{l}\text { Bloomfield-Zeisler, 1913; Brodsky, 2006; Broughton, } \\
\text { Stevens y Malloch, 2006; Bruser, 1997; Chenoll, 1990; } \\
\text { Colomer, 1990; Galamian, 1998; Gordon, 2003; Grindea, } \\
\text { 1991; Hoppenot, 2000; Juslin, 2003; Martenot, 1993; } \\
\text { Miklaszewski, 1989; Neuhaus, 1987; Palmer, 1997; } \\
\text { Parncutt, 2003; Vines, Krumhansl, Wanderley y Levitin, } \\
\text { 2006. }\end{array}$ \\
\hline $\begin{array}{l}\text { 18-Efectuar una práctica de riesgo, por medio de una } \\
\text { ejecución decidida y resuelta, comenzando firmemente } \\
\text { y evitando detenciones durante el período de } \\
\text { interpretación. }\end{array}$ & $\begin{array}{l}\text { Ballesteros, 2004; Bruser, 1997; Capistrán, 2017; } \\
\text { Chenoll, 1990; Cremaschi, 2012; Derbez, 2015; } \\
\text { Galamian, 1998; García-Rico, 2009; Gordon, 2003; } \\
\text { Mateo, 1997; Neuhaus, 1987; Pascuali y Príncipe, 1982; } \\
\text { Pin, 2001; Wrigley y Emmerson, 2013. }\end{array}$ \\
\hline $\begin{array}{l}\text { 19-Práctica consciente en la que el foco de atención se } \\
\text { sitúe en el presente de la ejecución, por medio de una } \\
\text { percepción sensorial dedicada por completo a la } \\
\text { actividad del momento interpretativo actual }\end{array}$ & $\begin{array}{l}\text { Bruser, 1997; Bunting, 1999; Chenoll, 1990; Coso, 1992; } \\
\text { Dalia y Pozo, 2006; Derbez, 2015; Hoppenot, 2000; } \\
\text { Oyan, 2006; Pérez Sánchez, 2003. }\end{array}$ \\
\hline $\begin{array}{l}\text { 20-Uso de mensajes constructivos de auto-motivación } \\
\text { para afianzar la confianza del propio intérprete, } \\
\text { transformando su miedo en valentía y/o llevando a cabo } \\
\text { un diálogo positivo consigo mismo. }\end{array}$ & $\begin{array}{l}\text { Arizcuren, 1985; Blum, 2000; Bruser, 1997; Chenoll, } \\
\text { 1990; Coso, 1992; Dalia y Pozo, 2006; Galper, 1989; } \\
\text { Hoppenot, 2000; Klöppel, 2005; Liertz, 2002; Manresa, } \\
\text { 2006; Michalak, 2006; Neuhaus, 1987; Ruiz, 1999; } \\
\text { Wilson y Roland, 2002. }\end{array}$ \\
\hline $\begin{array}{l}\text { 21-Manejo positivo de la crítica externa por medio de la } \\
\text { aceptación y desarrollo de las observaciones justas de } \\
\text { dicho juicio, en el margen de las posibilidades actuales }\end{array}$ & $\begin{array}{l}\text { Bruser, 1997; Dalia y Pozo, 2006; Gabrielsson, 2003; } \\
\text { García-Rico, 2010; Hoppenot, 2000; Kenny y } \\
\text { Ackermann, 2015; Klees-Dacheneder y Campo, 2000; }\end{array}$ \\
\hline
\end{tabular}

THE CONTENT OF THIS ARTICLE IS THE SOLE RESPONSIBILITY OF THE AUTHORS. THE REVISTA ELECTRÓNICA DE LEEME AND UNIVERSITAT DE VALĖNCIA ARE NOT LIABLE FOR ANY LEGAL ACTIONS THAT MAY ARISE INVOLVING THE ARTICLE'S CONTENT. REVISTA ELECTRÓNICA DE LEEME -LISTA ELECTRÓNICA EUROPEA DE MÚSICA EN LA EDUCACIÓNHTTP://MUSICA.REDIRIS.ES.ISSN: 1575-9563 EDITORES: UNIVERSIDAD DE VALENCIA Y JESÚS TEJADA GIMÉNEZ. VISIBILIDAD DE ESTA REVISTA: EBSCO, CINDOC (CESIC), CITEFACTOR, COPAC, DIALNET, DICE (CSIC), DOAJ, E-REVISTAS (CSIC), EBSCO PREMIER, ERIH+, GALE CENGAGE LEARNING, IN-RECS, IRESIE, LATINDEX, MIAR, OCLC WORLDCAT, RESH, REDIB, RILM CORE JOURNALS, SUDOC, ULRICHS, ZEITSCHRIFTDATENBANK, ESTA REVISTA ESTÁ PUBLICADA CON EL APOYO INSTITUCIONAL DE REDIRIS-CONSEJO SUPERIOR DE INVESTIGACIONES CIENTIFICAS Y ES DE ACCESO LIBRE. 


\subsubsection{Elaboración del programa didáctico}

En la segunda fase, se elaboró el programa cuyos fundamentos didácticos se hacen eco de ideas extraídas de investigaciones educativas encuadradas en el marco de la Psicología Cognitiva y en un enfoque naturalista de las Ciencias de la Educación, concretamente en el paradigma mediacional configurado en la Didáctica. En el ámbito de la Psicología Cognitiva, se tuvieron presentes el constructivismo y el aprendizaje significativo (Coll, 1996; Pozo, 1989; Pozo y Monereo, 1999). La concepción constructivista del aprendizaje, abordada por Torrado, Casas y Pozo (2005) en el ámbito musical, sitúa la actividad mental constructiva del estudiante en la base de los procesos de desarrollo personal que promueve la educación. Se consideran tres aspectos esenciales en la manera de entender el aprendizaje: aprendizaje significativo, memorización comprensiva y funcionalidad de lo aprendido.

Según Ausubel, Novak y Hanesian (1983), un aprendizaje es significativo cuando puede relacionarse, de manera no arbitraria y sustancial, con lo que el estudiante ya sabe. De modo que, un aprendizaje resulta significativo cuando puede integrarse a las estructuras de conocimiento que posee el individuo, es decir, cuando el nuevo material adquiere significado para el sujeto gracias a su relación con conocimientos previos (Pozo, 1989). El programa didáctico generó especial énfasis en que el aprendizaje de las estrategias de práctica instrumental se originara desde conocimientos previos, necesidades e intereses del propio sujeto, realizándose un tratamiento integrado y cíclico de las mismas, utilizando situaciones reales y propiciando un aprendizaje activo, constructivo y autónomo.

A su vez, también se tuvo en consideración la zona de desarrollo próximo de Vigotsky (1986), que explica el desfase existente entre la resolución individual y la social, de problemas y tareas cognitivas.

Asimismo, se adoptaron consideraciones de la teoría constructivista aplicada a la enseñanza de instrumentos musicales propuesta por Torrado et al. (2005). La enseñanza estuvo centrada en la interacción docente-estudiante, que conjuntamente controlan un determinado instrumento para producir la obra musical de la forma deseada. Se trató de fomentar la reflexión del futuro instrumentista sobre su propia práctica como principal vía para favorecer su comprensión, y con ello, impulsar una vía hacia la construcción de conocimientos en el marco de un aprendizaje, progresivamente, más autónomo (Torrado et al., 2005). La meta docente es que fuera el mismo estudiante el que guiara su propio aprendizaje; no se trataba de dirigirle externamente, sino de enseñarle a regular metacognitivamente su propia acción. Para ello, fue preciso un programa didáctico que propiciara una construcción conjunta en la que el instrumentista, con la ayuda del docente, lograra comprender el significado de la partitura, consiguiera interiorizar lo que deseaba transmitir, así como las estrategias que necesitaba

THE CONTENT OF THIS ARTICLE IS THE SOLE RESPONSIBILITY OF THE AUTHORS. THE REVISTA ELECTRÓNICA DE LEEME AND UNIVERSITAT DE VALĖNCIA ARE NOT LIABLE FOR ANY LEGAL ACTIONS THAT MAY ARISE INVOLVING THE ARTICLE'S CONTENT. REVISTA ELECTRÓNICA DE LEEME -LISTA ELECTRÓNICA EUROPEA DE MÚSICA EN LA EDUCACIÓNHTTP://MUSICA.REDIRIS.ES.ISSN: 1575-9563 EDITORES: UNIVERSIDAD DE VALENCIA Y JESÚS TEJADA GIMÉNEZ. VISIBILIDAD DE ESTA REVISTA: EBSCO, CINDOC (CESIC), CITEFACTOR, COPAC, DIALNET, DICE (CSIC), DOAJ, E-REVISTAS (CSIC), EBSCO PREMIER, ERIH+, GALE CENGAGE LEARNING, IN-RECS, IRESIE, LATINDEX, MIAR, OCLC WORLDCAT, RESH, REDIB, RILM CORE JOURNALS, SUDOC, ULRICHS, ZEITSCHRIFTDATENBANK, ESTA REVISTA ESTÁ PUBLICADA CON EL APOYO INSTITUCIONAL DE REDIRIS-CONSEJO SUPERIOR DE INVESTIGACIONES CIENTIFICAS Y ES DE ACCESO LIBRE. 
manejar para su óptima consecución. En definitiva, un modelo de enseñanza en el que la partitura y el instrumento sean el medio, pero no el fin del aprendizaje (Torrado et al., 2005).

Bajo esta concepción constructivista, se comprende la estrecha relación entre conocimiento y acción, de manera que únicamente podrá lograrse la regulación de la actividad motora de los instrumentistas desde su propio cuerpo y a partir de la propia reflexión del estudiante sobre sus estados mentales, percepciones, sentimientos y acciones (Torrado et al., 2005). Se trata de promover en el instrumentista una actividad metacognitiva en la que el estudiante que está aprendiendo a interpretar genere sus propias representaciones (cogniciones $\mathrm{y}$, también, emociones), reflexione sobre su propia acción y, de este modo, logre reconstruirla.

Tras las aportaciones de la Psicología Cognitiva, corresponde ahora tratar el ámbito de las Ciencias de la Educación. El programa didáctico fundamenta también su intervención didáctica en los presupuestos del paradigma mediacional, en sus dos vertientes: estudiante y docente. En cuanto al paradigma centrado en el estudiante, se adoptan principios referentes a su papel activo en función de sus elaboraciones y construcciones personales, siempre partiendo de sus conocimientos previos, necesidades... y por medio de un desafío cognitivo. Estos conocimientos previos pueden ser el resultado de experiencias educativas anteriores, académicas o no académicas, o de aprendizajes espontáneos (procesos cognitivos que se activan en el proceso didáctico) (Pérez Gómez, 1983). En la elaboración del programa, se tuvieron presentes las capacidades del estudiante, como actitudes o cualidades que disponen para el buen ejercicio, en los cinco ámbitos del desarrollo de Sánchez Huete (2008): cognitivas, motrices, afectivo-éticas, de comunicación y de actuación.

A tenor de los postulados del cognitivismo y de los establecidos desde el paradigma mediacional centrado en el estudiante, el rol docente está delimitado por las propuestas que emanan del paradigma mediacional centrado en su persona. La enseñanza es considerada como un proceso de planificación y ejecución de actuaciones, es decir, como un proceso de adopción de decisiones. Asimismo, la enseñanza se concibe como un proceso tecnológico de resolución de problemas. Un paso complejo de planificación racional de actos en un medio multidimensional, flexible y versátil que no admite comportamientos estándar ni estilos docentes prefijados (Pérez Gómez, 1983). En cada momento, el docente verifica los estados naturales y los procesos de transición de un estado a otro, supervisa la marcha de los diseños planificados y la eficacia de las estrategias manejadas. De algún modo, está permanentemente juzgando la situación, procesando y tomando decisiones, orientando la acción en base a dichas decisiones y vigilando el efecto de las acciones en los estudiantes (Pérez Gómez, 1983).

A partir de un análisis previo de necesidades y teniendo en cuenta todas estas premisas, se elaboró un programa didáctico de baja intensidad. Un programa que se desarrolla sin la imposición de consignas y en el contexto natural o habitual para los usuarios del mismo, utilizando actividades espontáneas o cotidianas (Anguera, 2008). En su elaboración, se tuvieron en cuenta los objetivos, el contexto y los recursos, procediéndose después a la puesta en marcha

THE CONTENT OF THIS ARTICLE IS THE SOLE RESPONSIBILITY OF THE AUTHORS. THE REVISTA ELECTRÓNICA DE LEEME AND UNIVERSITAT DE VALĖNCIA ARE NOT LIABLE FOR ANY LEGAL ACTIONS THAT MAY ARISE INVOLVING THE ARTICLE'S CONTENT. REVISTA ELECTRÓNICA DE LEEME -LISTA ELECTRÓNICA EUROPEA DE MÚSICA EN LA EDUCACIÓN.HTTP://MUSICA.REDIRIS.ES.ISSN: 1575-9563 EDITORES: UNIVERSIDAD DE VALENCIA Y JESÚS TEJADA GIMÉNEZ. VISIBILIDAD DE ESTA REVISTA: EBSCO, CINDOC (CESIC), CITEFACTOR, COPAC, DIALNET, DICE (CSIC), DOAJ, E-REVISTAS (CSIC), EBSCO PREMIER, ERIH+, GALE CENGAGE LEARNING, IN-RECS, IRESIE, LATINDEX, MIAR, OCLC WORLDCAT, RESH, REDIB, RILM CORE JOURNALS, SUDOC, ULRICHS, ZEITSCHRIFTDATENBANK, ESTA REVISTA ESTÁ PUBLICADA CON EL APOYO INSTITUCIONAL DE REDIRIS-CONSEJO SUPERIOR DE INVESTIGACIONES CIENTIFICAS Y ES DE ACCESO LIBRE. 
de dicho programa y efectuándose procesualmente una evaluación formativa a lo largo del feedback establecido entre las distintas fases del proceso (Anguera, 2008).

\subsubsection{Puesta en práctica del programa didáctico}

En una tercera fase de la investigación, se implementó el programa didáctico sobre las estrategias de práctica instrumental identificadas, en el que participaron 28 estudiantes de 17 especialidades instrumentales diferentes. Cada instrumentista recibió cinco sesiones de implementación del programa didáctico, de una hora de duración cada una, que fueron convenientemente grabadas en vídeo y en las que se puso en práctica cada una de las 21 estrategias. En la Figura 1, puede observarse un esquema del desarrollo del mismo:

INTERPRETACIÓN INICIAL DE LA OBRA MUSICAL

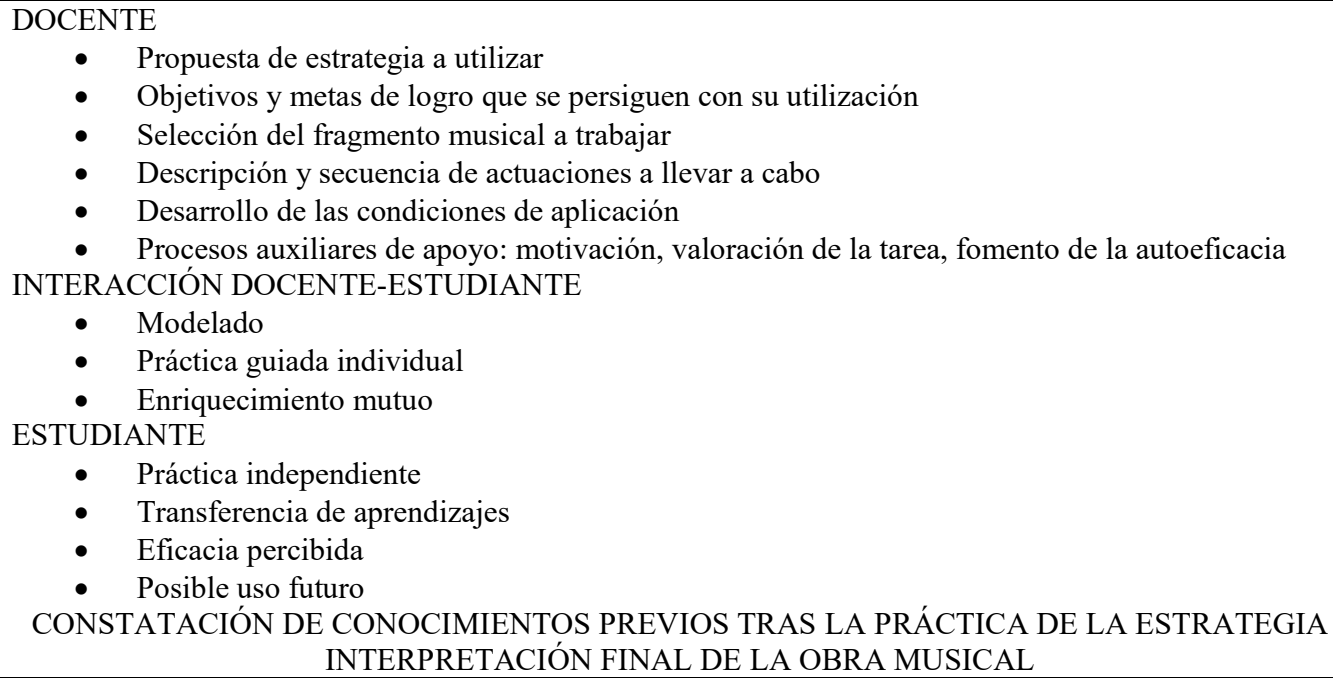

- Propuesta de estrategia a utilizar

- Objetivos y metas de logro que se persiguen con su utilización

- Selección del fragmento musical a trabajar

- Descripción y secuencia de actuaciones a llevar a cabo

- Desarrollo de las condiciones de aplicación

- Procesos auxiliares de apoyo: motivación, valoración de la tarea, fomento de la autoeficacia INTERACCIÓN DOCENTE-ESTUDIANTE

- Modelado

- Práctica guiada individual

- Enriquecimiento mutuo

ESTUDIANTE

- Práctica independiente

- Transferencia de aprendizajes

- Eficacia percibida

- $\quad$ Posible uso futuro

CONSTATACIÓN DE CONOCIMIENTOS PREVIOS TRAS LA PRÁCTICA DE LA ESTRATEGIA INTERPRETACIÓN FINAL DE LA OBRA MUSICAL

Fig. 1. Implementación del programa didáctico de estrategias de práctica instrumental

\subsection{Instrumentos y técnicas de recogida de datos}

Tras la puesta en práctica de cada una de las estrategias del programa, se llevó a cabo la breve entrevista semiestructurada que principalmente se caracterizó por su flexibilidad, permitiéndose posibles variaciones en la secuencia establecida, así como en la formulación. Su objetivo era recabar información detallada acerca del conocimiento previo y el posible uso anterior de cada una de las estrategias de práctica instrumental trabajadas en el programa didáctico.

THE CONTENT OF THIS ARTICLE IS THE SOLE RESPONSIBILITY OF THE AUTHORS. THE REVISTA ELECTRÓNICA DE LEEME AND UNIVERSITAT DE VALĖNCIA ARE NOT LIABLE FOR ANY LEGAL ACTIONS THAT MAY ARISE INVOLVING THE ARTICLE'S CONTENT. REVISTA ELECTRÓNICA DE LEEME -LISTA ELECTRÓNICA EUROPEA DE MÚSICA EN LA EDUCACIÓN.HTTP://MUSICA.REDIRIS.ES.ISSN: 1575-9563 EDITORES: UNIVERSIDAD DE VALENCIA Y JESÚS TEJADA GIMÉNEZ. VISIBILIDAD DE ESTA REVISTA: EBSCO, CINDOC (CESIC), CITEFACTOR, COPAC, DIALNET, DICE (CSIC), DOAJ, E-REVISTAS (CSIC), EBSCO PREMIER, ERIH+, GALE CENGAGE LEARNING, IN-RECS, IRESIE, LATINDEX, MIAR, OCLC WORLDCAT, RESH, REDIB, RILM CORE JOURNALS, SUDOC, ULRICHS, ZEITSCHRIFTDATENBANK, ESTA REVISTA ESTÁ PUBLICADA CON EL APOYO INSTITUCIONAL DE REDIRIS-CONSEJO SUPERIOR DE INVESTIGACIONES CIENTIFICAS Y ES DE ACCESO LIBRE. 
Para la elaboración del protocolo de la entrevista, se tuvo presente los ítems que configurarían el cuestionario (instrumento mencionado en la fase posterior), de modo que fuese posible establecer una relación y contraste entre los datos obtenidos mediante ambos instrumentos. El protocolo de la entrevista fue sometido a juicio de expertos con objeto de validar su comprensibilidad y objetividad y, una vez realizadas dos vueltas de prueba piloto de la misma para detectar posibles deficiencias, se elaboró el diseño final de la misma. Posteriormente, se llevó a cabo su aplicación y tratamiento de datos, mediante el sistema categorial y su codificación.

En la cuarta fase, y gracias a un cuestionario final, se recopiló información de los estudiantes una semana después de su participación en el programa didáctico. Dicho cuestionario se fundamentó en los conocimientos previos acerca de cada estrategia de práctica instrumental y permitió abordar la amplia muestra de participantes garantizando su anonimato.

A la hora de realizar el cuestionario se tuvieron presentes diversos aspectos:

- $\quad$ Objetivos: en primer lugar, servir a los objetivos de la investigación.

- Mantener la atención del encuestado: para ello se intentó conseguir un discurso ordenado en el cuestionario de modo que al estudiante le resultara difícil perder la continuidad.

- $\quad$ Lenguaje empleado: se utilizó un lenguaje que fuera fácilmente comprensible por los estudiantes participantes.

- $\quad$ Evitar sesgos: en la elaboración del cuestionario se trató de mostrar objetividad en el planteamiento de las preguntas de modo que no se fomentara la elección de una determinada respuesta.

- $\quad$ Extensión del cuestionario: se trató de realizar un cuestionario breve y conciso, con las preguntas precisas para lograr los objetivos previstos.

En un primer momento, se elaboró un cuestionario provisional y mediante el sistema de jueces se validó su comprensibilidad, la no directividad de las preguntas, así como la univocidad y objetividad de sus preguntas. Una vez obtenidas las opiniones de los jueces expertos y confeccionada la segunda versión del mismo, se procedió a obtener el índice de fiabilidad del mismo (Alfa de Cronbach de .909). Para ello, se realizó el cuestionario a una muestra reducida (10 participantes no pertenecientes a la muestra seleccionada); pero, sí a una población con características similares a los sujetos a estudio y se estimó el nivel de fiabilidad de la información recogida mediante dicho instrumento.

Finalmente, se efectuó la triangulación de los diferentes resultados, llevándose a cabo un análisis descriptivo de los datos obtenidos en la entrevista y de aquellos obtenidos mediante el cuestionario final relativos a la situación de partida de los participantes con respecto a cada

THE CONTENT OF THIS ARTICLE IS THE SOLE RESPONSIBILITY OF THE AUTHORS. THE REVISTA ELECTRÓNICA DE LEEME AND UNIVERSITAT DE VALĖNCIA ARE NOT LIABLE FOR ANY LEGAL ACTIONS THAT MAY ARISE INVOLVING THE ARTICLE'S CONTENT. REVISTA ELECTRÓNICA DE LEEME -LISTA ELECTRÓNICA EUROPEA DE MÚSICA EN LA EDUCACIÓN.HTTP://MUSICA.REDIRIS.ES.ISSN: 1575-9563 EDITORES: UNIVERSIDAD DE VALENCIA Y JESÚS TEJADA GIMÉNEZ. VISIBILIDAD DE ESTA REVISTA: EBSCO, CINDOC (CESIC), CITEFACTOR, COPAC, DIALNET, DICE (CSIC), DOAJ, E-REVISTAS (CSIC), EBSCO PREMIER, ERIH+, GALE CENGAGE LEARNING, IN-RECS, IRESIE, LATINDEX, MIAR, OCLC WORLDCAT, RESH, REDIB, RILM CORE JOURNALS, SUDOC, ULRICHS, ZEITSCHRIFTDATENBANK, ESTA REVISTA ESTÁ PUBLICADA CON EL APOYO INSTITUCIONAL DE REDIRIS-CONSEJO SUPERIOR DE INVESTIGACIONES CIENTIFICAS Y ES DE ACCESO LIBRE. 
una de las estrategias de práctica instrumental. De este modo, el procedimiento de triangulación ha permitido obtener un mayor control de calidad en el proceso de investigación y, según Aguilar y Barroso (2015), supone una garantía de los resultados alcanzados en lo referente a su validez, credibilidad y rigor.

\section{Resultados}

En el presente trabajo, se estudió la situación de partida de los estudiantes de instrumento con respecto al contenido del programa didáctico; ya que, en principio, cabría pensar que, al ingresar en Grado Superior de Música tras aproximadamente 10 años de estudios musicales, deberían tener un manejo adecuado de la mayor parte de las estrategias de práctica de acreditada eficacia. Sin embargo, los datos obtenidos mediante el cuestionario evidencian que, de media, tan sólo el $38.89 \%$ de los estudiantes conocían y manejaban las propuestas del programa didáctico antes de su participación. En un análisis pormenorizado en la Figura 2, se muestran las diversas estrategias ordenadas según el porcentaje de instrumentistas que las conocían y utilizaban en su práctica habitual antes de su participación en el programa didáctico:

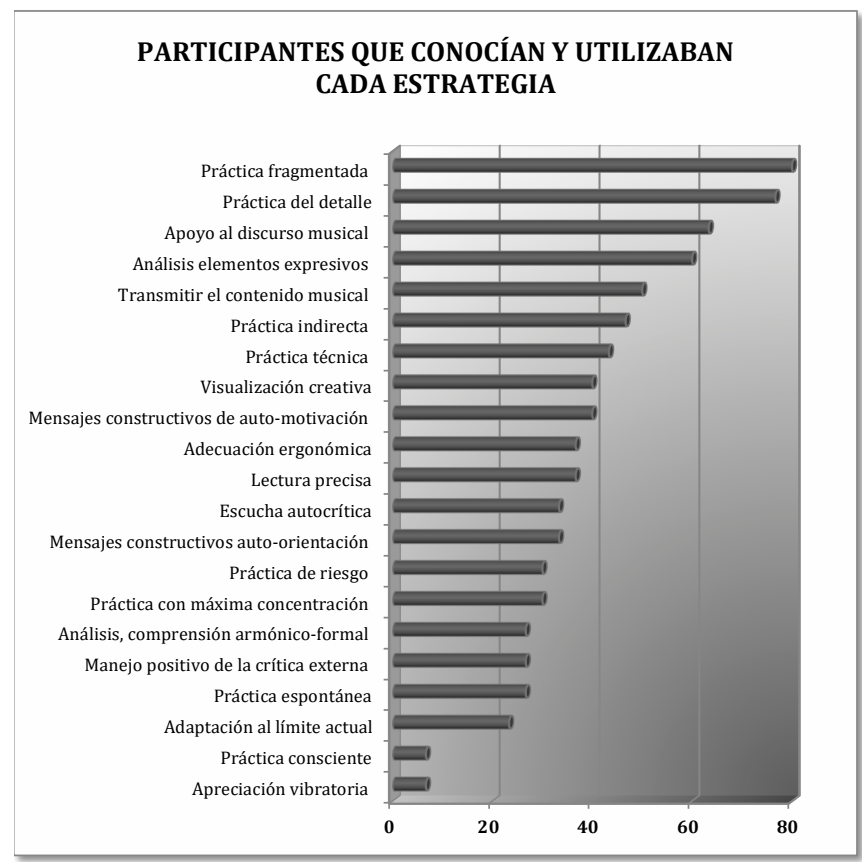

Fig. 2. Porcentaje de estudiantes que conocían y manejaban una determinada estrategia antes de participar en el programa didáctico

Según la Figura 2, la estrategia de práctica fragmentada y la práctica del detalle fueron las propuestas más manejadas en la práctica habitual de los instrumentistas antes de participar

THE CONTENT OF THIS ARTICLE IS THE SOLE RESPONSIBILITY OF THE AUTHORS. THE REVISTA ELECTRÓNICA DE LEEME AND UNIVERSITAT DE VALĖNCIA ARE NOT LIABLE FOR ANY LEGAL ACTIONS THAT MAY ARISE INVOLVING THE ARTICLE'S CONTENT. REVISTA ELECTRÓNICA DE LEEME -LISTA ELECTRÓNICA EUROPEA DE MÚSICA EN LA EDUCACIÓNHTTP://MUSICA.REDIRIS.ES.ISSN: 1575-9563 EDITORES: UNIVERSIDAD DE VALENCIA Y JESÚS TEJADA GIMÉNEZ. VISIBILIDAD DE ESTA REVISTA: EBSCO, CINDOC (CESIC), CITEFACTOR, COPAC, DIALNET, DICE (CSIC), DOAJ, E-REVISTAS (CSIC), EBSCO PREMIER, ERIH+, GALE CENGAGE LEARNING, IN-RECS, IRESIE, LATINDEX, MIAR, OCLC WORLDCAT, RESH, REDIB, RILM CORE JOURNALS, SUDOC, ULRICHS, ZEITSCHRIFTDATENBANK, ESTA REVISTA ESTÁ PUBLICADA CON EL APOYO INSTITUCIONAL DE REDIRIS-CONSEJO SUPERIOR DE INVESTIGACIONES CIENTIFICAS Y ES DE ACCESO LIBRE. 
en el programa didáctico. De hecho, en las entrevistas se verifica que son consideradas unas estrategias básicas en la formación instrumental. Muchos de los participantes pueden analizar al detalle cada una de ellas y la mayor parte de ellos asegura haber recibido formación sobre las mismas desde los comienzos de su aprendizaje con el instrumento. Así mismo, en la Figura 3 y 4 pueden consultarse los datos del cuestionario acerca de las estrategias de práctica conocidas y utilizadas previamente según cada familia de instrumentistas.

\section{Leyenda:}

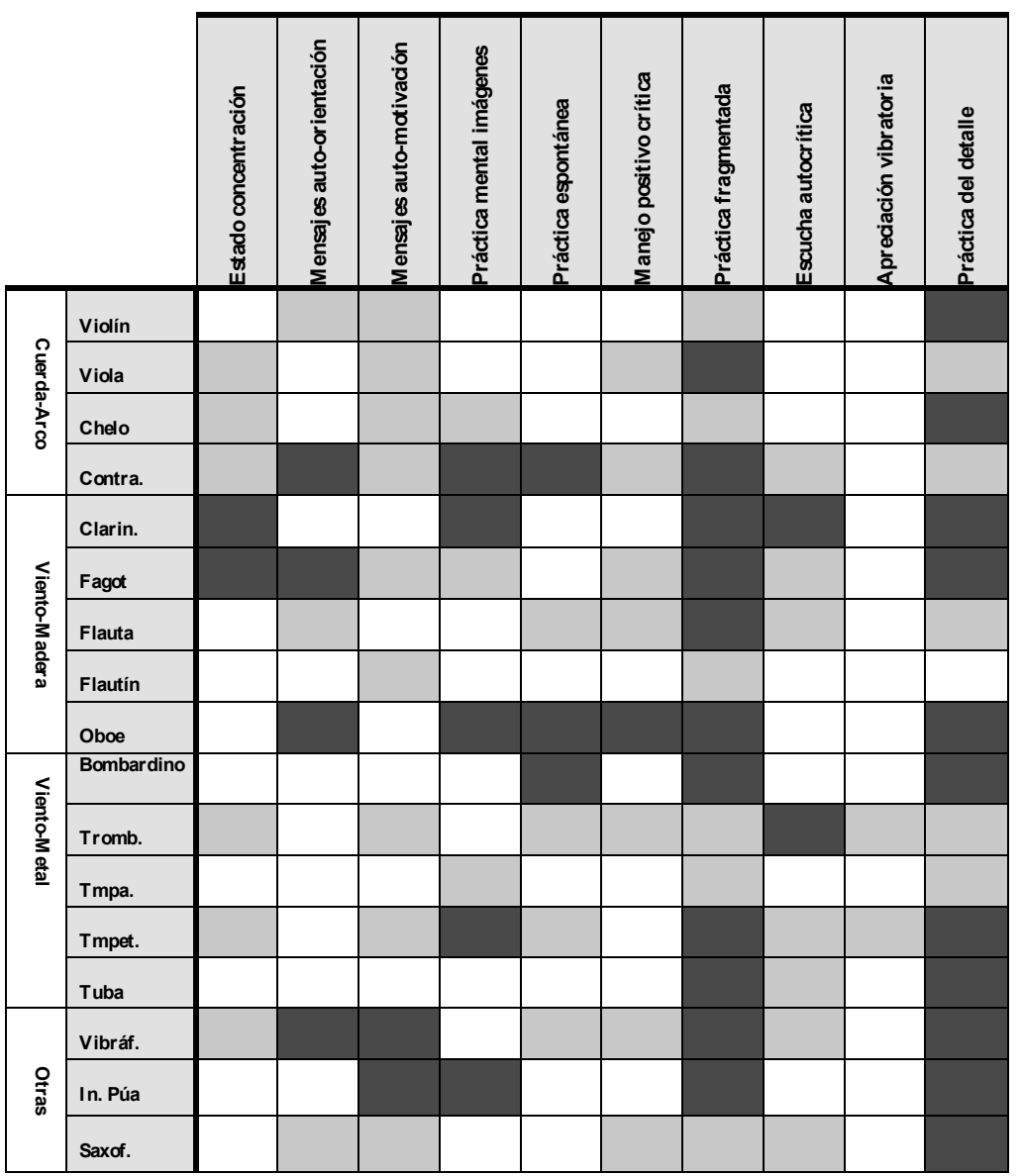

Fig. 3. Estrategias de práctica instrumental conocidas y utilizadas previamente según cada instrumento

THE CONTENT OF THIS ARTICLE IS THE SOLE RESPONSIBILITY OF THE AUTHORS. THE REVISTA ELECTRÓNICA DE LEEME AND UNIVERSITAT DE VALĖNCIA ARE NOT LIABLE FOR ANY LEGAL ACTIONS THAT MAY ARISE INVOLVING THE ARTICLE'S CONTENT. REVISTA ELECTRÓNICA DE LEEME -LISTA ELECTRÓNICA EUROPEA DE MÚSICA EN LA EDUCACIÓNHTTP://MUSICA.REDIRIS.ES.ISSN: 1575-9563 EDITORES: UNIVERSIDAD DE VALENCIA Y JESÚS TEJADA GIMÉNEZ. VISIBILIDAD DE ESTA REVISTA: EBSCO, CINDOC (CESIC), CITEFACTOR, COPAC, DIALNET, DICE (CSIC), DOAJ, E-REVISTAS (CSIC), EBSCO PREMIER, ERIH+, GALE CENGAGE LEARNING, IN-RECS, IRESIE, LATINDEX, MIAR, OCLC WORLDCAT, RESH, REDIB, RILM CORE JOURNALS, SUDOC, ULRICHS, ZEITSCHRIFTDATENBANK, ESTA REVISTA ESTÁ PUBLICADA CON EL APOYO INSTITUCIONAL DE REDIRIS-CONSEJO SUPERIOR DE INVESTIGACIONES CIENTIFICAS Y ES DE ACCESO LIBRE. 


\section{Leyenda:}

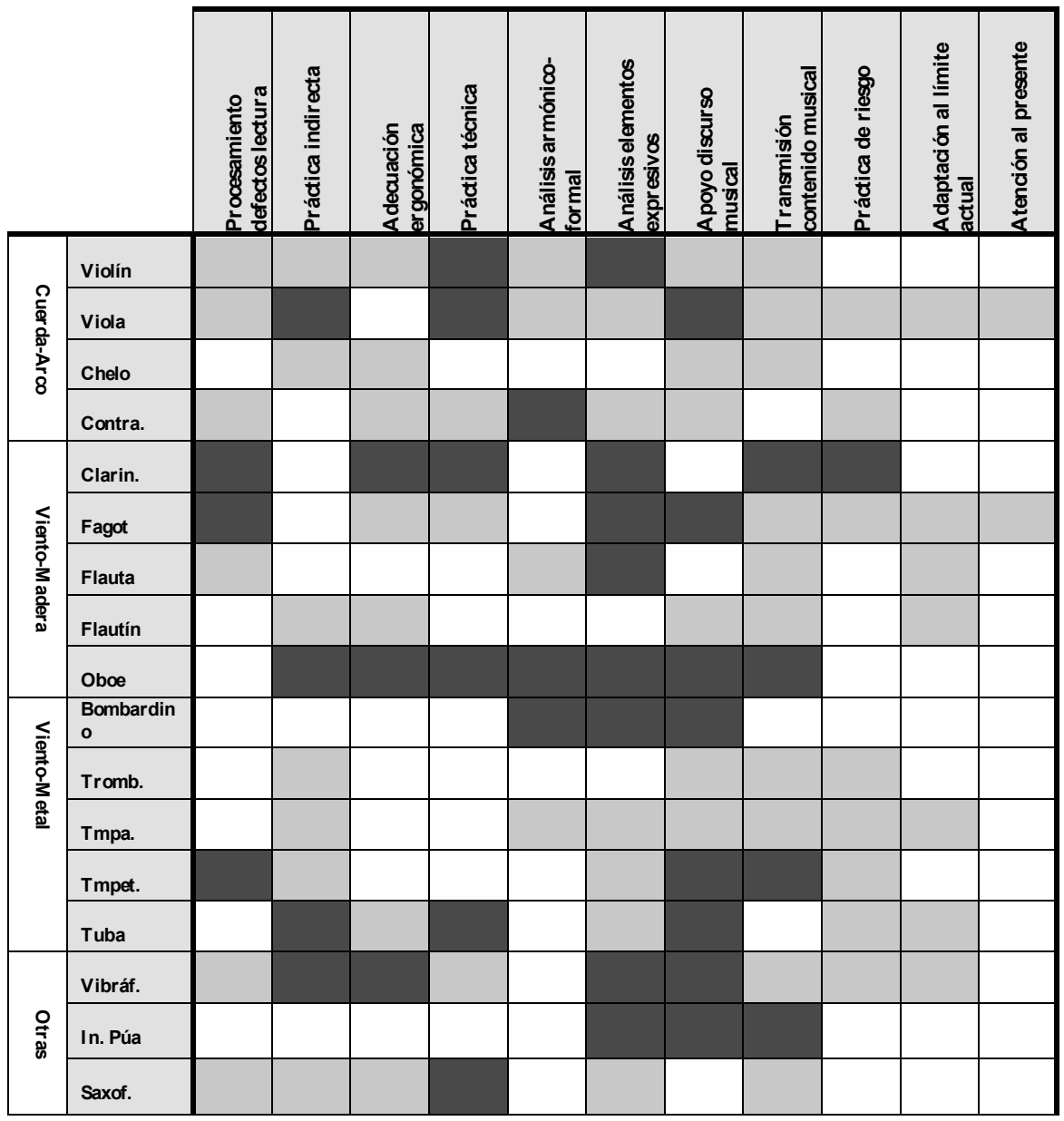

Fig. 4. Estrategias de práctica instrumental conocidas y utilizadas previamente según cada instrumento

A este respecto, destacan los participantes de viento-madera por ser, según los datos del cuestionario, los que menos necesitarían participar en el programa didáctico por conocer previamente un mayor número de las estrategias del mismo. A su vez, en las entrevistas se corrobora que los instrumentistas de viento-madera conocen al detalle del desarrollo de las mismas y aseguran haber recibido formación sobre las estrategias desde los comienzos de su aprendizaje instrumental.

THE CONTENT OF THIS ARTICLE IS THE SOLE RESPONSIBILITY OF THE AUTHORS. THE REVISTA ELECTRÓNICA DE LEEME AND UNIVERSITAT DE VALĖNCIA ARE NOT LIABLE FOR ANY LEGAL ACTIONS THAT MAY ARISE INVOLVING THE ARTICLE'S CONTENT. REVISTA ELECTRÓNICA DE LEEME -LISTA ELECTRÓNICA EUROPEA DE MÚSICA EN LA EDUCACIÓNHTTP://MUSICA.REDIRIS.ES.ISSN: 1575-9563 EDITORES: UNIVERSIDAD DE VALENCIA Y JESÚS TEJADA GIMÉNEZ. VISIBILIDAD DE ESTA REVISTA: EBSCO, CINDOC (CESIC), CITEFACTOR, COPAC, DIALNET, DICE (CSIC), DOAJ, E-REVISTAS (CSIC), EBSCO PREMIER, ERIH+, GALE CENGAGE LEARNING, IN-RECS, IRESIE, LATINDEX, MIAR, OCLC WORLDCAT, RESH, REDIB, RILM CORE JOURNALS, SUDOC, ULRICHS, ZEITSCHRIFTDATENBANK, ESTA REVISTA ESTÁ PUBLICADA CON EL APOYO INSTITUCIONAL DE REDIRIS-CONSEJO SUPERIOR DE INVESTIGACIONES CIENTIFICAS Y ES DE ACCESO LIBRE. 
Por otra parte, y según los datos proporcionados extraídos por el cuestionario, en la Figura 5 se refleja el porcentaje de participantes que desconocía una determinada estrategia de práctica instrumental antes de participar en el programa didáctico:

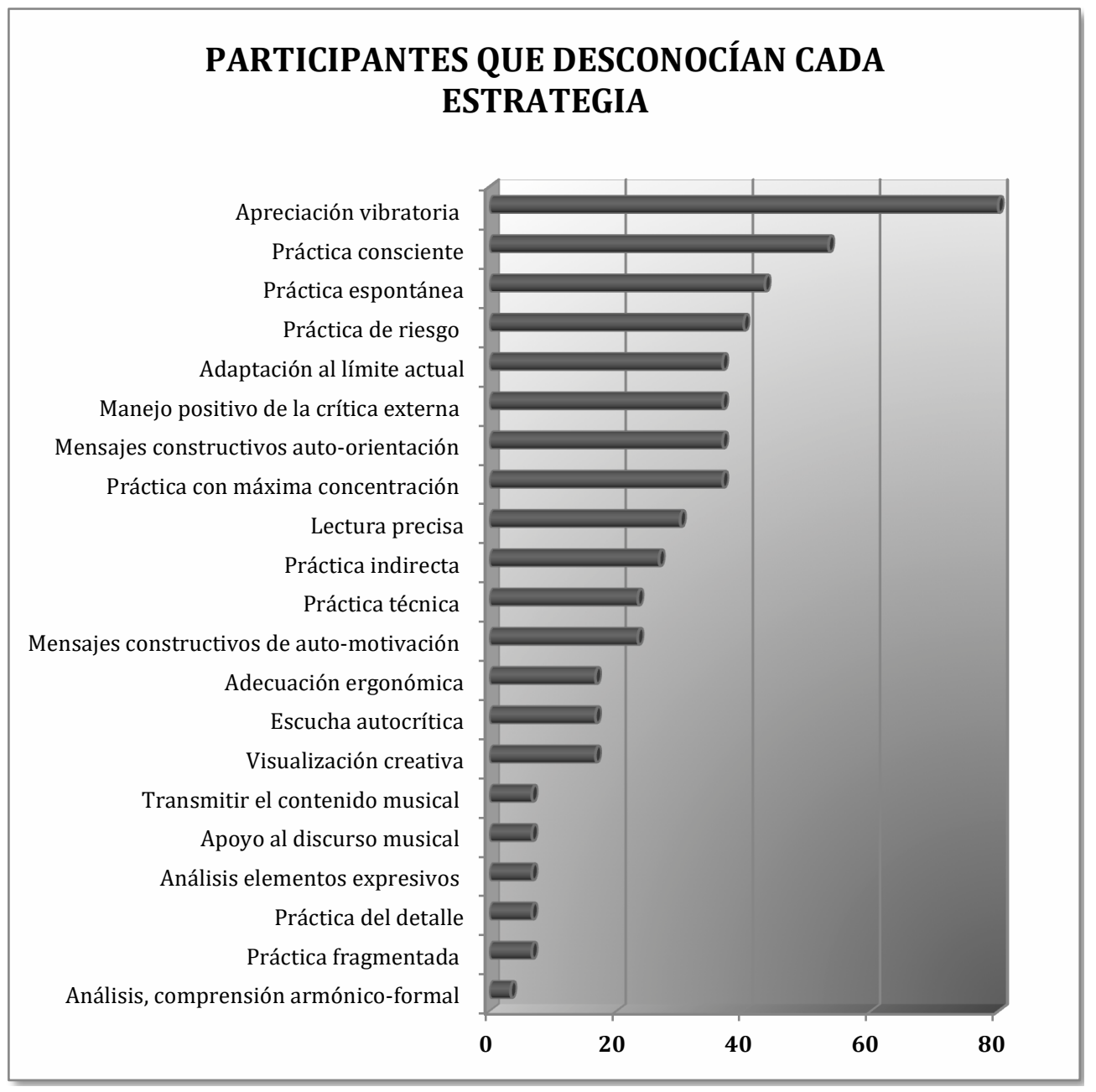

Fig. 5. Porcentaje de estudiantes que desconocía una determinada estrategia antes de participar en el programa didáctico

Según la Figura 5, la estrategia de apreciación vibratoria y la de práctica consciente eran las más desconocidas por los estudiantes antes de participar en el programa didáctico. De hecho, en las entrevistas muestran su extrañeza y dudan de la utilidad de la estrategia de apreciación vibratoria. En cuanto a la práctica consciente, los estudiantes mayoritariamente manifiestan conocer dicha estrategia, aunque señalan que todavía no forma parte de su práctica habitual. Esto implica que, generalmente, saben lo que tienen que hacer durante la práctica deliberada de

THE CONTENT OF THIS ARTICLE IS THE SOLE RESPONSIBILITY OF THE AUTHORS. THE REVISTA ELECTRÓNICA DE LEEME AND UNIVERSITAT DE VALĖNCIA ARE NOT LIABLE FOR ANY LEGAL ACTIONS THAT MAY ARISE INVOLVING THE ARTICLE'S CONTENT. REVISTA ELECTRÓNICA DE LEEME -LISTA ELECTRÓNICA EUROPEA DE MÚSICA EN LA EDUCACIÓN.HTTP://MUSICA.REDIRIS.ES.ISSN: 1575-9563 EDITORES: UNIVERSIDAD DE VALENCIA Y JESÚS TEJADA GIMÉNEZ. VISIBILIDAD DE ESTA REVISTA: EBSCO, CINDOC (CESIC), CITEFACTOR, COPAC, DIALNET, DICE (CSIC), DOAJ, E-REVISTAS (CSIC), EBSCO PREMIER, ERIH+, GALE CENGAGE LEARNING, IN-RECS, IRESIE, LATINDEX, MIAR, OCLC WORLDCAT, RESH, REDIB, RILM CORE JOURNALS, SUDOC, ULRICHS, ZEITSCHRIFTDATENBANK, ESTA REVISTA ESTÁ PUBLICADA CON EL APOYO INSTITUCIONAL DE REDIRIS-CONSEJO SUPERIOR DE INVESTIGACIONES CIENTIFICAS Y ES DE ACCESO LIBRE. 
su instrumento a nivel teórico, pero no necesariamente lo efectúan en su estudio real diario. Sin embargo, son conscientes de su importancia sobre todo desde su acceso al Conservatorio Superior. En la Figura 6 y 7, se pueden consultar las estrategias no conocidas por cada familia de instrumentistas antes de participar en el programa didáctico, según los datos revelados por el cuestionario final:

\section{Leyenda:}

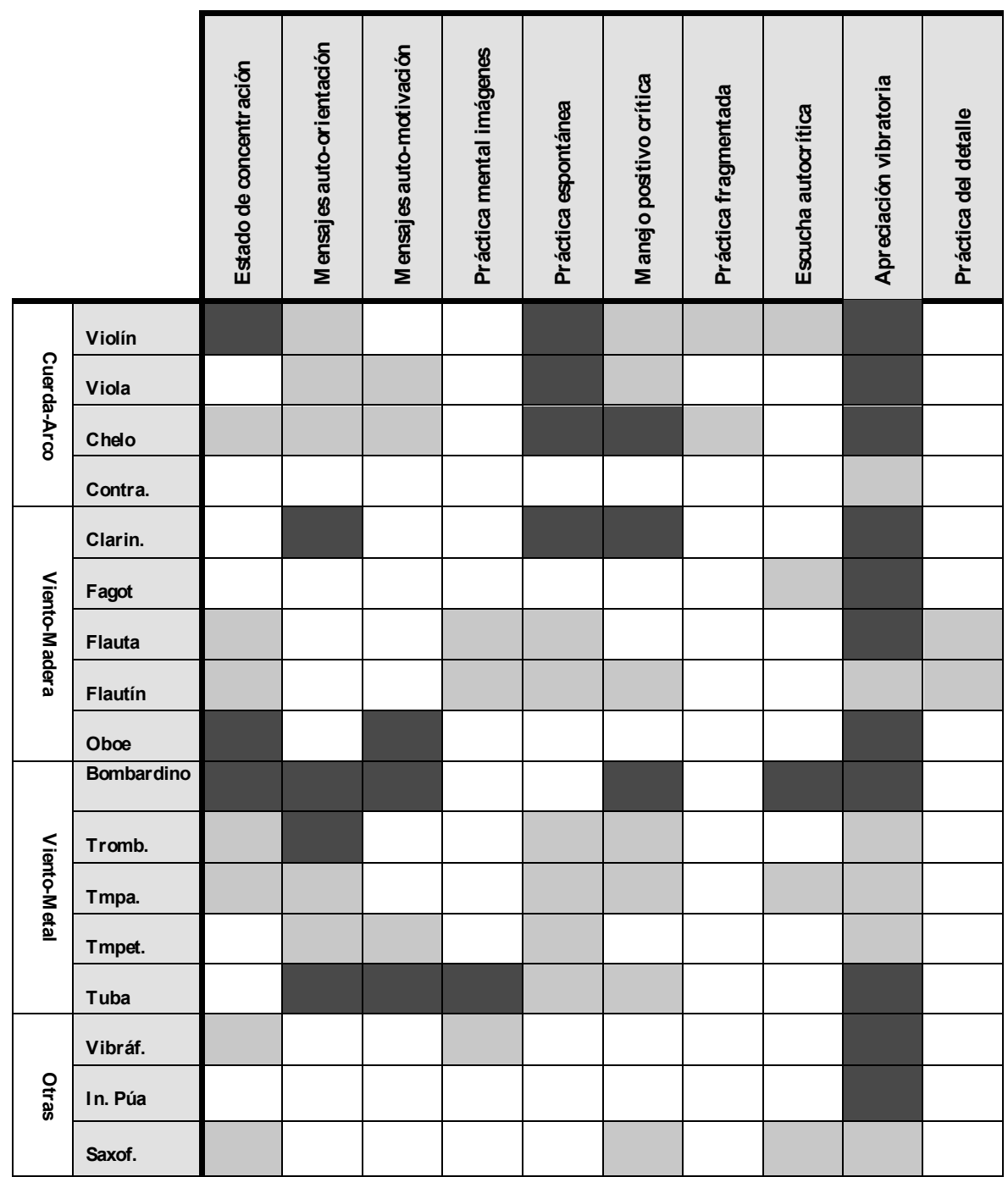

Fig. 6. Estrategias de práctica instrumental que eran desconocidas antes de participar en el programa didáctico según el instrumento del estudiante

THE CONTENT OF THIS ARTICLE IS THE SOLE RESPONSIBILITY OF THE AUTHORS. THE REVISTA ELECTRÓNICA DE LEEME AND UNIVERSITAT DE VALĖNCIA ARE NOT LIABLE FOR ANY LEGAL ACTIONS THAT MAY ARISE INVOLVING THE ARTICLE'S CONTENT. REVISTA ELECTRÓNICA DE LEEME -LISTA ELECTRÓNICA EUROPEA DE MÚSICA EN LA EDUCACIÓNHTTP://MUSICA.REDIRIS.ES.ISSN: 1575-9563 EDITORES: UNIVERSIDAD DE VALENCIA Y JESÚS TEJADA GIMÉNEZ. VISIBILIDAD DE ESTA REVISTA: EBSCO, CINDOC (CESIC), CITEFACTOR, COPAC, DIALNET, DICE (CSIC), DOAJ, E-REVISTAS (CSIC), EBSCO PREMIER, ERIH+, GALE CENGAGE LEARNING, IN-RECS, IRESIE, LATINDEX, MIAR, OCLC WORLDCAT, RESH, REDIB, RILM CORE JOURNALS, SUDOC, ULRICHS, ZEITSCHRIFTDATENBANK, ESTA REVISTA ESTA PUBLICADA CON EL APOYO INSTITUCIONAL DE REDIRIS-CONSEJO SUPERIOR DE INVESTIGACIONES CIENTIFICAS Y ES DE ACCESO LIBRE. 


\section{Leyenda:}

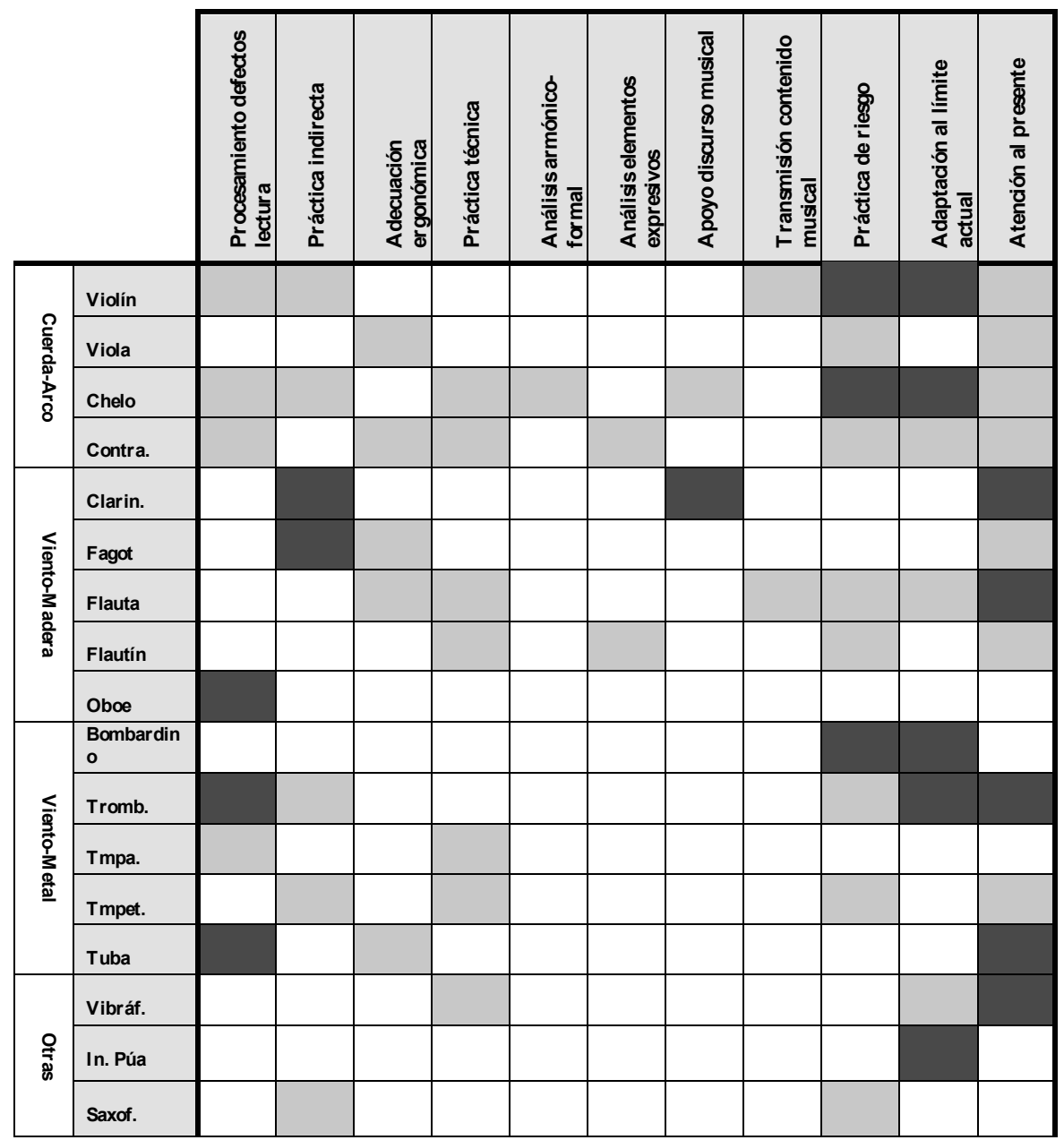

Fig. 7. Estrategias de práctica instrumental que eran desconocidas antes de participar en el programa didáctico según el instrumento del estudiante

En este sentido, cabe destacar que los instrumentistas de viento-metal son los que, según el cuestionario, más desconocen las propuestas trabajadas. De hecho, en las entrevistas aseguran que son los que más necesitan participar en el programa didáctico ya que creen tener un menor conocimiento previo acerca de estrategias de estudio. Este hecho es atribuido,

THE CONTENT OF THIS ARTICLE IS THE SOLE RESPONSIBILITY OF THE AUTHORS. THE REVISTA ELECTRÓNICA DE LEEME AND UNIVERSITAT DE VALĖNCIA ARE NOT LIABLE FOR ANY LEGAL ACTIONS THAT MAY ARISE INVOLVING THE ARTICLE'S CONTENT. REVISTA ELECTRÓNICA DE LEEME -LISTA ELECTRÓNICA EUROPEA DE MÚSICA EN LA EDUCACIÓN.HTTP://MUSICA.REDIRIS.ES.ISSN: 1575-9563 EDITORES: UNIVERSIDAD DE VALENCIA Y JESÚS TEJADA GIMÉNEZ. VISIBILIDAD DE ESTA REVISTA: EBSCO, CINDOC (CESIC), CITEFACTOR, COPAC, DIALNET, DICE (CSIC), DOAJ, E-REVISTAS (CSIC), EBSCO PREMIER, ERIH+, GALE CENGAGE LEARNING, IN-RECS, IRESIE, LATINDEX, MIAR, OCLC WORLDCAT, RESH, REDIB, RILM CORE JOURNALS, SUDOC, ULRICHS, ZEITSCHRIFTDATENBANK, ESTA REVISTA ESTÁ PUBLICADA CON EL APOYO INSTITUCIONAL DE REDIRIS-CONSEJO SUPERIOR DE INVESTIGACIONES CIENTIFICAS Y ES DE ACCESO LIBRE. 
principalmente, a la falta de bibliografía sobre el tema relativa a los instrumentos de vientometal.

\section{Discusión}

En principio, es posible pensar que un estudiante de instrumento que accede al Grado Superior de Música, debería tener una práctica efectiva y estratégica. Sin embargo, la experiencia docente permite comprobar que no es así en numerosos casos; en ocasiones el estudiante no conoce las estrategias, o bien pese a conocerlas no forman parte de su práctica habitual.

De modo que, en cuanto a la situación de partida de los estudiantes, se ha podido constatar que los contenidos del programa didáctico implementado eran, en general, poco conocidos. Varios estudios ya habían puesto en evidencia que los profesionales poseen un conocimiento limitado de estrategias específicas de práctica instrumental (Hallam, 2001; Pitts et al., 2002; Renwick y McPherson, 2002). A vez, se ha puesto de manifiesto que pueden ser conocidas; pero, sin embargo, ser poco utilizadas. Byo y Cassidy (2008) ya sugirieron que los estudiantes generalmente saben lo que tienen que hacer durante el estudio individual de su instrumento, pero no necesariamente lo efectúan en su práctica habitual.

En este estudio, se sugiere la poca relevancia que han tenido las estrategias de práctica instrumental durante el periodo de formación previo, es decir, durante las enseñanzas profesionales de Música. De hecho, durante 3 años consecutivos, Jørgensen (2004) solicitó información a los estudiantes de nuevo ingreso en el Conservatorio Superior de Noruega, al igual que en el presente estudio. Y sus resultados evidencian que aproximadamente el $40 \%$ de los encuestados respondió que su profesorado anterior había puesto muy poco o ningún énfasis en las estrategias de estudio instrumental.

Probablemente la explicación a este hecho esté en que su desarrollo no es necesariamente sencillo. En el estudio de Barry y McArthur (1994), la mayoría de los docentes encuestados afirmaron que siempre o casi siempre discutían la importancia de la práctica y sus estrategias específicas con los estudiantes. Sin embargo, puede darse el caso de que lo que el estudiante recuerde no corresponda exactamente con lo que el docente considera que ha comunicado. De hecho, Kostka (2002) observó la discrepancia entre lo que los docentes creen enseñar sobre la práctica instrumental y lo que realmente aprenden los estudiantes sobre la misma. No obstante, como cabría esperar, la estrategia de práctica fragmentada ha sido la más conocida y utilizada previamente por los instrumentistas, según los resultados de la presente investigación y según las conclusiones del estudio de Gruson (1988) y las de Miklaszewski (1989). Resulta comprensible, ya que es una propuesta ampliamente empleada en el ámbito de la docencia desde el inicio de la formación instrumental y que, tanto progenitores como entrenadores repiten multitud de veces durante el desarrollo de un individuo.

THE CONTENT OF THIS ARTICLE IS THE SOLE RESPONSIBILITY OF THE AUTHORS. THE REVISTA ELECTRÓNICA DE LEEME AND UNIVERSITAT DE VALĖNCIA ARE NOT LIABLE FOR ANY LEGAL ACTIONS THAT MAY ARISE INVOLVING THE ARTICLE'S CONTENT. REVISTA ELECTRÓNICA DE LEEME -LISTA ELECTRÓNICA EUROPEA DE MÚSICA EN LA EDUCACIÓNHTTP://MUSICA.REDIRIS.ES.ISSN: 1575-9563 EDITORES: UNIVERSIDAD DE VALENCIA Y JESÚS TEJADA GIMÉNEZ. VISIBILIDAD DE ESTA REVISTA: EBSCO, CINDOC (CESIC), CITEFACTOR, COPAC, DIALNET, DICE (CSIC), DOAJ, E-REVISTAS (CSIC), EBSCO PREMIER, ERIH+, GALE CENGAGE LEARNING, IN-RECS, IRESIE, LATINDEX, MIAR, OCLC WORLDCAT, RESH, REDIB, RILM CORE JOURNALS, SUDOC, ULRICHS, ZEITSCHRIFTDATENBANK, ESTA REVISTA ESTÁ PUBLICADA CON EL APOYO INSTITUCIONAL DE REDIRIS-CONSEJO SUPERIOR DE INVESTIGACIONES CIENTIFICAS Y ES DE ACCESO LIBRE. 
Por el contrario, la estrategia de apreciación vibratoria y la de práctica consciente han sido las más desconocidas por los instrumentistas antes de participar en dicho programa. Los resultados también son comprensibles, ya que el hecho de experimentar la transmisión de vibraciones varía mucho en función del instrumento musical utilizado.

Por familias instrumentales, los resultados obtenidos también resultan de interés. Los instrumentistas de viento-metal, que son los que probablemente posean menos bibliografía relativa a los procesos de enseñanza-aprendizaje de sus respectivos instrumentos, son los que más desconocían las estrategias de práctica instrumental antes de probarlas en el transcurso del programa didáctico llevado a cabo durante el estudio.

Para finalizar el capítulo de discusión, a modo de conclusión, se detallan los siguientes puntos recopilatorios:

1. Se evidencia que los estudiantes que acceden a Grado Superior poseen un bagaje de estrategias de práctica instrumental insuficiente, tal y como habían constatado estudios anteriores (Byo y Cassidy, 2008; Hallam, 2001; Pitts et al., 2002; Renwick y McPherson, 2002), así como un conocimiento limitado de las mismas que varía en función del instrumento estudiado, lo que justifica la necesidad de implementar un programa didáctico sobre estrategias de práctica.

2. Cualquier actuación estratégica del instrumentista requiere saber, poder y querer aprender a aprender, igual que defienden Torrado et al. (2005), mientras implica una importante toma de decisiones en la que se movilizan los motivos, actitudes, conceptos y procedimientos necesarios en función de cada situación específica de aprendizaje musical.

3. Las estrategias de práctica instrumental se sitúan en un plano fundamentalmente interdisciplinar e incluso transdisciplinar, en la medida en que ponen en juego la planificación y regulación consciente de acciones, pensamientos, pautas de conducta... dirigidas a una meta musical, superando el plano de lo meramente académico e incidiendo directamente en el plano profesional, como ya sugirió con anterioridad Jørgensen (2004).

4. El estudio resalta la necesidad de establecer un conocimiento sólidamente fundamentado acerca del ensayo, que oriente y motive a los futuros músicos de la forma más adecuada, puesto que la verdadera meta de la docencia instrumental debería ser, enseñar al estudiante cómo practicar para que adquiera su autonomía como intérprete.

5. Se sugiere que el programa didáctico resulta muy provechoso para convertir las clases de formación instrumental en sesiones de práctica eficaz de tal manera que el docente, en lugar de limitarse a exponer los puntos fuertes y débiles de la interpretación de su alumnado, sea capaz de involucrarse en su modo de estudio.

THE CONTENT OF THIS ARTICLE IS THE SOLE RESPONSIBILITY OF THE AUTHORS. THE REVISTA ELECTRÓNICA DE LEEME AND UNIVERSITAT DE VALĖNCIA ARE NOT LIABLE FOR ANY LEGAL ACTIONS THAT MAY ARISE INVOLVING THE ARTICLE'S CONTENT. REVISTA ELECTRÓNICA DE LEEME -LISTA ELECTRÓNICA EUROPEA DE MÚSICA EN LA EDUCACIÓN.HTTP://MUSICA.REDIRIS.ES.ISSN: 1575-9563 EDITORES: UNIVERSIDAD DE VALENCIA Y JESÚS TEJADA GIMÉNEZ. VISIBILIDAD DE ESTA REVISTA: EBSCO, CINDOC (CESIC), CITEFACTOR, COPAC, DIALNET, DICE (CSIC), DOAJ, E-REVISTAS (CSIC), EBSCO PREMIER, ERIH+, GALE CENGAGE LEARNING, IN-RECS, IRESIE, LATINDEX, MIAR, OCLC WORLDCAT, RESH, REDIB, RILM CORE JOURNALS, SUDOC, ULRICHS, ZEITSCHRIFTDATENBANK, ESTA REVISTA ESTA PUBLICADA CON EL APOYO INSTITUCIONAL DE REDIRIS-CONSEJO SUPERIOR DE INVESTIGACIONES CIENTIFICAS Y ES DE ACCESO LIBRE. 
De modo que, esta investigación saca a la luz lo beneficioso que resulta que el docente se responsabilice de supervisar los ensayos de sus instrumentistas y de generar oportunidades de aplicación de práctica efectiva, deliberada e intensa en el aula adecuadas a cada sujeto y en función de su nivel de destreza. En definitiva, no hay que olvidar que los resultados obtenidos alertan acerca de la necesidad de formar a los estudiantes de Grado Superior en la práctica estratégica con objeto de optimizar su estudio individual.

Por otra parte, la principal limitación del estudio ha sido que la muestra abordada ha pretendido describir la situación de un entorno concreto como es el Conservatorio Superior de Música de Aragón por lo que no se pueden generalizar los resultados a lo que puede ocurrir en otros centros estatales o de ámbito internacional, ni los objetivos del estudio pretenden ahondar en una posible explicación de los hechos. No obstante, el tema objeto de estudio presenta distintas líneas de actuación por lo que cada una de ellas abre nuevas y variadas vías para investigaciones posteriores.

Una primera vía de interés radica en el proceso de incorporación de las diversas estrategias de práctica en función del desarrollo evolutivo del estudiante de instrumento ya que, probablemente, sus posibilidades de manejo difieran en función de si el usuario de las mismas se trata de un niño, un joven o de un adulto.

La segunda vía implica diseñar una investigación a largo plazo para comprender cómo el estudiante incorpora nuevas estrategias a su práctica habitual y cómo optimiza sus ensayos y mejora su rendimiento a nivel escénico, es decir, durante la actuación pública.

Otra de las vías de interés se fundamenta en la observación de sesiones de práctica deliberada e intensa de grandes intérpretes de las diferentes especialidades instrumentales, para comprender con detalle cómo manejan las diversas estrategias de práctica instrumental, si pueden identificarse nuevas estrategias, cuáles son las más requeridas y cómo evolucionan en función de la fase de estudio en la que se encuentra la obra musical.

En definitiva, se han trazado novedosas y variadas líneas para la continuidad investigadora, de modo que deseo fervientemente que cualquier interesado que consulte este trabajo, descubra algún aspecto que le sea de utilidad para conseguir su propósito, al igual que pueda encontrar la dirección adecuada en caso contrario.

THE CONTENT OF THIS ARTICLE IS THE SOLE RESPONSIBILITY OF THE AUTHORS. THE REVISTA ELECTRÓNICA DE LEEME AND UNIVERSITAT DE VALĖNCIA ARE NOT LIABLE FOR ANY LEGAL ACTIONS THAT MAY ARISE INVOLVING THE ARTICLE'S CONTENT. REVISTA ELECTRÓNICA DE LEEME -LISTA ELECTRÓNICA EUROPEA DE MÚSICA EN LA EDUCACIÓN.HTTP://MUSICA.REDIRIS.ES.ISSN: 1575-9563 EDITORES: UNIVERSIDAD DE VALENCIA Y JESÚS TEJADA GIMÉNEZ. VISIBILIDAD DE ESTA REVISTA: EBSCO, CINDOC (CESIC), CITEFACTOR, COPAC, DIALNET, DICE (CSIC), DOAJ, E-REVISTAS (CSIC), EBSCO PREMIER, ERIH+, GALE CENGAGE LEARNING, IN-RECS, IRESIE, LATINDEX, MIAR, OCLC WORLDCAT, RESH, REDIB, RILM CORE JOURNALS, SUDOC, ULRICHS, ZEITSCHRIFTDATENBANK, ESTA REVISTA ESTÁ PUBLICADA CON EL APOYO INSTITUCIONAL DE REDIRIS-CONSEJO SUPERIOR DE INVESTIGACIONES CIENTIFICAS Y ES DE ACCESO LIBRE. 
LISTA ELECTRÓNICA EUROPEA DE MÚSICA EN LA EDUCACIÓN

\section{Referencias}

Aguilar, S., y Barroso, J. (2015). La triangulación de datos como estrategia en investigación educativa. Píxel-Bit. Revista de Medios y Educación, 47, 73-88.

Altenmüller, E. y Gruhn, W. (2002). Brain mechanisms. En R. Parncutt y G. E. McPherson (Eds.), The Science and Psychology of Music Performance: Creative Strategies for Teaching and Learning (pp. 63-81). Oxford: Oxford University Press.

Anguera, M. T. (2008). Diseños evaluativos de baja intervención. En M. T. Anguera, S. Chacón y A. Blanco (Coords.), Evaluación de Programas Sociales y Sanitarios (pp. 153-184). Madrid: Síntesis.

Arizcuren, E. (1985). Técnica del violonchelo. Principios básicos. Madrid: Centro de publicaciones. Ministerio de Educación y Ciencia.

Artaud, P. I. (1991). La flauta. Barcelona: Editorial Labor.

Ausubel, D. P., Novak, J. D. y Hanesian, H. (1983). Psicología educativa: un punto de vista cognoscitivo. México: Trillas.

Bachmann, M. L. (1998). La rítmica Jaques-Dalcroze. Una educación por la música y para la música. Madrid: Ediciones Pirámide.

Ballesteros, M. (2004). El contrabajo. Una visión integral. Oviedo: Editorial Trabe.

Barrie, H. (2007). An organist's "How to practise". Toronto: Royal Canadian College of Organists National Standing Committee for Publications.

Barry, N. H. (1990). The effects of different practice techniques upon technical accuracy and musicality in student instrumental music performance. Research Perspectives in Music Education, 1, 4-8.

Barry, N. H. y Hallam, S. (2002). Practice. En R. Parncutt y G. E. McPherson (Eds.), The Science and Psychology of Music Performance: Creative Strategies for Teaching and Learning (pp. 151-165). Oxford: Oxford University Press.

Barry, N. H., y McArthur, V. (1994). Teaching practice strategies in the music studio: A survey of applied music teachers. Psychology of Music, 22, 44-55.

Bauer, H. (1913). Artistic aspects of piano study. En J. F. Cooke (Ed.), Great Pianists on Piano Playing (pp. 64-79). Philadelphia: Theo. Presser.

Bengtsson, S. L., Nagy, Z., Skare, S., Forsman, L., Forssberg, H. y Ullén, F. (2005). Extensive piano practicing has regionally specific effects on white matter development. Nature Neuroscience, 8 (9), 1148-1150.

THE CONTENT OF THIS ARTICLE IS THE SOLE RESPONSIBILITY OF THE AUTHORS. THE REVISTA ELECTRÓNICA DE LEEME AND UNIVERSITAT DE VALĖNCIA ARE NOT LIABLE FOR ANY LEGAL ACTIONS THAT MAY ARISE INVOLVING THE ARTICLE'S CONTENT. REVISTA ELECTRÓNICA DE LEEME -LISTA ELECTRÓNICA EUROPEA DE MÚSICA EN LA EDUCACIÓN.HTTP://MUSICA.REDIRIS.ES.ISSN: 1575-9563 EDITORES: UNIVERSIDAD DE VALENCIA Y JESÚS TEJADA GIMÉNEZ. VISIBILIDAD DE ESTA REVISTA: EBSCO, CINDOC (CESIC), CITEFACTOR, COPAC, DIALNET, DICE (CSIC), DOAJ, E-REVISTAS (CSIC), EBSCO PREMIER, ERIH+, GALE CENGAGE LEARNING, IN-RECS, IRESIE, LATINDEX, MIAR, OCLC WORLDCAT, RESH, REDIB, RILM CORE JOURNALS, SUDOC, ULRICHS, ZEITSCHRIFTDATENBANK, ESTA REVISTA ESTÁ PUBLICADA CON EL APOYO INSTITUCIONAL DE REDIRIS-CONSEJO SUPERIOR DE INVESTIGACIONES CIENTIFICAS Y ES DE ACCESO LIBRE. 
Bernardi, N. F., Schories, A., Jabusch, H. C., Colombo, B. y Altenmüller, E. (2009). Mental practice in music memorization: an ecological-empirical study. En J. Louhivuori, T. Eerola, S. Saarikallio, T. Himberg y P. Eerola (Eds.), Proceedings of the 7th Triennial Conference of European Society for the Cognitive Sciences of Music (pp. 20-27). Jyväskylä, Finland: ESCOM.

Besson, M. y Schön, D. (2003). Comparison between language and music. En I. Peretz. y R. J. Zatorre (Eds.), The Cognitive Neuroscience of Music (pp. 269-293). Oxford: Oxford University Press.

Bigand, E. y Pineau, M. (1997). Global context effects on musical expectancy. Perception y Psychophysics, 59 (7), 1098-1107.

Bloomfield-Zeisler, F. (1913). Appearing in public. En J. F. Cooke (Ed.), Great Pianists on Piano Playing (pp. 80-96). Philadelphia: Theo. Presser.

Blum, D. (2000). El arte del cuarteto de cuerda. El cuarteto Guarneri en conversación con David Blum. Barcelona: Idea Books.

Boehm, T. (1847). La flauta y la interpretación flautística. Madrid: Ed. Mundimúsica.

Brodsky, W. (2006). In the wings of British orchestras: A multi-episode interview study among symphony players. Journal of Occupational and Organizational Psychology, 79 (4), 673-690.

Broughton, M. y Stevens, C. (2009). Physical movement and imagery in professional and undergraduate student solo marimba practice. En A. Williamon, Pretty, S. y Buck, R. (Eds.), Proceedings of the International Symposium on Performance Science 2009 (pp. 531-536). Utrecht, The Netherlands: European Association of Conservatoires (AEC).

Broughton, M., Stevens, C. y Malloch, S (2006). Music, movement and marimba: An investigation of the role of movement and gesture in communicating musical expression to an audience. En M. Baroni, A. R. Addessi, R. Caterina y M. Costa (Eds.), Proceedings of the 9th International Conference on Music Perception and Cognition (pp. 1127-1135). Bolonia: Alma Mater Studiorum University of Bologna.

Bruser, M. (1997). The art of practicing. A guide to making music from the heart. New York: Three Rivers Press.

Bunting, C. (1999). El arte de tocar el violonchelo. Madrid: Ediciones Pirámide.

Burwell, K. y Shipton, M. (2013). Strategic Approaches to practice: an action research project. British Journal of Music Education, 30 (03), 329-345.

Byo, J. y Cassidy, J. W. (2008). An exploratory study of time use in the practice of music majors self-report and observation analysis. Update: Applications of Research in Music Education, 27 (1), 33-40.

THE CONTENT OF THIS ARTICLE IS THE SOLE RESPONSIBILITY OF THE AUTHORS. THE REVISTA ELECTRÓNICA DE LEEME AND UNIVERSITAT DE VALĖNCIA ARE NOT LIABLE FOR ANY LEGAL ACTIONS THAT MAY ARISE INVOLVING THE ARTICLE'S CONTENT. REVISTA ELECTRÓNICA DE LEEME -LISTA ELECTRÓNICA EUROPEA DE MÚSICA EN LA EDUCACIÓN.HTTP://MUSICA.REDIRIS.ES.ISSN: 1575-9563 EDITORES: UNIVERSIDAD DE VALENCIA Y JESÚS TEJADA GIMÉNEZ. VISIBILIDAD DE ESTA REVISTA: EBSCO, CINDOC (CESIC), CITEFACTOR, COPAC, DIALNET, DICE (CSIC), DOAJ, E-REVISTAS (CSIC), EBSCO PREMIER, ERIH+, GALE CENGAGE LEARNING, IN-RECS, IRESIE, LATINDEX, MIAR, OCLC WORLDCAT, RESH, REDIB, RILM CORE JOURNALS, SUDOC, ULRICHS, ZEITSCHRIFTDATENBANK, ESTA REVISTA ESTÁ PUBLICADA CON EL APOYO INSTITUCIONAL DE REDIRIS-CONSEJO SUPERIOR DE INVESTIGACIONES CIENTIFICAS Y ES DE ACCESO LIBRE. 
Calvo-Manzano, M. R. (1987). Tratado analítico de la técnica y estética del arpa. Madrid: Editorial Alpuerto.

Calvo-Manzano, M. R. (2008). La higiene postural: ¿Cómo conseguir la naturalidad en las técnicas instrumentales? Comunicación presentada en el Seminario Internacional de Investigación Educativa en Apreciación e Interpretación Musical, Madrid. Recuperado de http://seminarioucmpoveda08.blogspot.com

Capistrán, R. W. (2017). ¡No sé cómo practicar! Propuesta metodológica para la práctica instrumental efectiva. ARTSEDUCA, 16, 8-31.

Carlevaro, A. (2000). Escuela de la guitarra. Exposición de la teoría instrumental. Buenos Aires: Ed. Barry.

Casas, A. (2013). Culturas de aprendizaje musical: concepciones, procesos y prácticas de aprendizaje en Clásico, Flamenco y Jazz. Madrid: Universidad autónoma de Madrid.

Chafin, R. y Imreh, G. (1997). "Pulling teeth and torture": musical memory and problem solving. Thinking y Reasoning, 3 (4), 315-336.

Chafin, R. y Imreh, G. (2001). A comparison of practice and self-report as sources of information about the goals of expert practice. Psychology of Music, 29, 39-69.

Chaffin, R., Imreh, G. y Crawford, M. E. (2002). Coda. En R. Chaffin, G. Imreh y M. E. Crawford (Eds.), Practicing perfection: Memory and piano performance (pp. 247-269). Mahwah, New Jersey: Lawrence Erlbaum.

Chaffin, R., Imreh, G., Lemieux, A. F. y Chen, C. (2003). "Seeing the big picture": Piano practice as expert problem solving. Music Perception, 20 (4), 465-490.

Chaffin, R. y Lemieux, A. F. (2004). General perspectives on achieving musical excellence. En A. Williamon (Ed.), Musical excellence. Strategies and techniques to enhance performance (pp.19-39). London: Oxford University Press.

Chaffin, R., Lisboa, T., Logan, T. y Begosh, K. T. (2010). Preparing for memorized cello performance: The role of performance cues. Psychology of Music, 38 (1), 3-30.

Chenoll, J. (1990). El trombón. Su historia. Su técnica. Madrid: Real Musical.

Clark, T. y Williamon, A. (2009). Imaging the music: A context-specific method for assessing imagery ability. En A. Williamon, S. Pretty y R. Buck (Eds.), Proceedings of the International Symposium on Performance Science 2009 (pp. 573-578). Utrecht, The Netherlands: European Association of Conservatoires (AEC).

THE CONTENT OF THIS ARTICLE IS THE SOLE RESPONSIBILITY OF THE AUTHORS. THE REVISTA ELECTRÓNICA DE LEEME AND UNIVERSITAT DE VALĖNCIA ARE NOT LIABLE FOR ANY LEGAL ACTIONS THAT MAY ARISE INVOLVING THE ARTICLE'S CONTENT. REVISTA ELECTRÓNICA DE LEEME -LISTA ELECTRÓNICA EUROPEA DE MÚSICA EN LA EDUCACIÓNHTTP://MUSICA.REDIRIS.ES.ISSN: 1575-9563 EDITORES: UNIVERSIDAD DE VALENCIA Y JESÚS TEJADA GIMÉNEZ. VISIBILIDAD DE ESTA REVISTA: EBSCO, CINDOC (CESIC), CITEFACTOR, COPAC, DIALNET, DICE (CSIC), DOAJ, E-REVISTAS (CSIC), EBSCO PREMIER, ERIH+, GALE CENGAGE LEARNING, IN-RECS, IRESIE, LATINDEX, MIAR, OCLC WORLDCAT, RESH, REDIB, RILM CORE JOURNALS, SUDOC, ULRICHS, ZEITSCHRIFTDATENBANK, ESTA REVISTA ESTÁ PUBLICADA CON EL APOYO INSTITUCIONAL DE REDIRIS-CONSEJO SUPERIOR DE INVESTIGACIONES CIENTIFICAS Y ES DE ACCESO LIBRE. 
Clarke, E. F. (1982). Timing in the performance of Erik Satie's 'Vexations'. Acta Psychologica, 50, 1-19.

Clarke, E. F. (1988). Generative principles in music performance. En J. A. Sloboda (Ed.), Generative processes in music: the psychology of performance, improvisation, and composition (pp. 1-26). Oxford: Clarendon Press.

Coll, C. (1996). Aprendizaje escolar y construcción del conocimiento. Barcelona: Ediciones Paidos.

Colomer, C. (1990). Sobre interpretación pianística. Madrid: Editorial Alpuerto.

Connolly, C. y Williamon, A. (2004). Mental skills training. En A. Williamon (Ed.), Musical excellence. Strategies and techniques to enhance performance (pp.221-245). London: Oxford University Press.

Coso, J. A. (1992). Tocar un instrumento. Metodología del estudio. Psicología y experiencia educativa en el aprendizaje instrumental. Madrid: Editorial Música Mundana.

Coyle, D. (2009). Las claves del talento. Barcelona: Ed. Planeta.

Cremaschi, A. (2012). The efect of a practice checklist on practice strategies, practice selfregulation and achievement of collegiate music majors enrolled in a beginning class piano course. Research Studies in Music Education, 34 (2), 223-233.

Csikszentmihalyi, M. (1990). Flow: The psychology of optimal experience. New York: HarperyRow.

Dalia, G. y Pozo, A. (2006). El músico. Una introducción a la psicología de la interpretación musical. Madrid: Mundimúsica ediciones.

Daniel, R. (2001). Self-assessment in performance. British Journal of Music Education, 18 (3), 215-226.

Davidson, J. W. (1993). Visual perception of performance manner in the movements of solo musicians. Psychology of Music, 21 (2), 103-113.

Derbez, P. (2015). El músico consciente. Editorial Endemus.

Dobson, M. C. (2010). Performing yourself? Autonomy and self-expression in the work of jazz musicians and classical string players. Music Performance Research, 3 (1), 42-60.

Driskell, J. E., Copper, C. y Moran, A. (1994). Does mental practice enhance performance? Journal of Applied Psychology, 79 (4), 481-492.

Dunsby, J. (1995). Performing music. Shared concerns. Oxford: Clarendon Press.

THE CONTENT OF THIS ARTICLE IS THE SOLE RESPONSIBILITY OF THE AUTHORS. THE REVISTA ELECTRÓNICA DE LEEME AND UNIVERSITAT DE VALĖNCIA ARE NOT LIABLE FOR ANY LEGAL ACTIONS THAT MAY ARISE INVOLVING THE ARTICLE'S CONTENT. REVISTA ELECTRÓNICA DE LEEME -LISTA ELECTRÓNICA EUROPEA DE MÚSICA EN LA EDUCACIÓN.HTTP://MUSICA.REDIRIS.ES.ISSN: 1575-9563 EDITORES: UNIVERSIDAD DE VALENCIA Y JESÚS TEJADA GIMÉNEZ. VISIBILIDAD DE ESTA REVISTA: EBSCO, CINDOC (CESIC), CITEFACTOR, COPAC, DIALNET, DICE (CSIC), DOAJ, E-REVISTAS (CSIC), EBSCO PREMIER, ERIH+, GALE CENGAGE LEARNING, IN-RECS, IRESIE, LATINDEX, MIAR, OCLC WORLDCAT, RESH, REDIB, RILM CORE JOURNALS, SUDOC, ULRICHS, ZEITSCHRIFTDATENBANK, ESTA REVISTA ESTÁ PUBLICADA CON EL APOYO INSTITUCIONAL DE REDIRIS-CONSEJO SUPERIOR DE INVESTIGACIONES CIENTIFICAS Y ES DE ACCESO LIBRE. 
Dyer, W. W. (1978). Tus zonas erróneas. Barcelona: Ed Grijalbo.

Eguilaz, M. J. (2009). La memoria en la interpretación guitarrística. Una aproximación a su problemática. Revista electrónica de LEEME, 23 (3). Recuperado de http://musica.rediris.es/leeme

Emond, B., Vinson, N. G., Singer, J., Barfurth, M. A. y Brooks, M. F. (2006). ReView: A digital video player to support music practice and learning. Journal of Technology in Music Learning, 1, 1-27.

Esteban, E. (2007). When gesture sounds: Bodily significance in musical performance. En A. Williamon y D. Coimbra (Eds.), Proceedings of the International Symposium on Performance Science 2007 (pp. 55-60). Utrecht, The Netherlands: European Association of Conservatoires (AEC).

Fields, R. D. (2006). Advances in understanding neuron-glia interactions. Neuron Glia Biology, $2(1), 23-26$.

Fields, R. D. (2008). White Matter Matters. Scientific American, 298, 54-61.

Foldes, A. (1992). Claves del teclado. Un libro para pianistas. Buenos Aires: Editorial Ricordi.

Friberg, A. y Sundberg, J. (1999). Does music performance allude to locomotion? A model of final ritardandi derived from measurements of stopping runners. The Journal of the Acoustical Society of America, 105 (3), 1469-1484.

Fujisawa, T., Iwami, N., Kinou, M. y Miura, M. (2009). Relationship between playing strategy and surface electromyograms in playing drums. En A. Williamon, S. Pretty y R. Buck (Eds.), Proceedings of the International Symposium on Performance Science 2009 (pp. 285-290). Utrecht, The Netherlands: European Association of Conservatoires (AEC).

Gabrielsson, A. (2003). Music performance research at the millennium. Psychology of music, 31 (3), 221-272.

Galamian, I. (1998). Interpretación y enseñanza del violín. Madrid: Ed. Pirámide.

García-Rico, J. (2009). Nicholas Angelich: "La música es algo vivo, no nos pertenece". Scherzo, 238, 138-142.

García-Rico, J. (2010). Entrevista a Javier Perianes. Scherzo, 251, 56-63.

Ginsborg, J. (2004). Strategies for memorizing music. En A. Williamon (Ed.), Musical excellence. Strategies and techniques to enhance performance (pp.122-141). London: Oxford University Press.

THE CONTENT OF THIS ARTICLE IS THE SOLE RESPONSIBILITY OF THE AUTHORS. THE REVISTA ELECTRÓNICA DE LEEME AND UNIVERSITAT DE VALĖNCIA ARE NOT LIABLE FOR ANY LEGAL ACTIONS THAT MAY ARISE INVOLVING THE ARTICLE'S CONTENT. REVISTA ELECTRÓNICA DE LEEME -LISTA ELECTRÓNICA EUROPEA DE MÚSICA EN LA EDUCACIÓN.HTTP://MUSICA.REDIRIS.ES.ISSN: 1575-9563 EDITORES: UNIVERSIDAD DE VALENCIA Y JESÚS TEJADA GIMÉNEZ. VISIBILIDAD DE ESTA REVISTA: EBSCO, CINDOC (CESIC), CITEFACTOR, COPAC, DIALNET, DICE (CSIC), DOAJ, E-REVISTAS (CSIC), EBSCO PREMIER, ERIH+, GALE CENGAGE LEARNING, IN-RECS, IRESIE, LATINDEX, MIAR, OCLC WORLDCAT, RESH, REDIB, RILM CORE JOURNALS, SUDOC, ULRICHS, ZEITSCHRIFTDATENBANK, ESTA REVISTA ESTÁ PUBLICADA CON EL APOYO INSTITUCIONAL DE REDIRIS-CONSEJO SUPERIOR DE INVESTIGACIONES CIENTIFICAS Y ES DE ACCESO LIBRE. 
Goebl, W., Dixon, S., De Poli, G., Friberg, A., Bresin, R. y Widmer, G. (2008). Sense in expressive music performance: Data acquisition, computational studies, and models. En P. Polotti y D. Rocchesso (Eds.), Sound to sense - sense to sound: A state of the art in sound and music computing (pp. 195-242). Berlin: Logos Verlag.

Goebl, W. y Palmer, C. (2008). Tactile feedback and timing accuracy in piano performance. Experimental Brain Research, 186 (3), 471-479.

Gordon, S. (2003). Técnicas maestras de piano. Lecciones magistrales de piano para estudiantes y profesores. Barcelona: Ediciones Robinbook.

Grindea, C. (1991). La tensión en el intérprete. Algunos consejos antes y durante la ejecución. Música y Educación: Revista internacional de pedagogía musical, 4 (7), 9-18.

Gruson, L. M. (1988). Rehearsal skill and musical competence: does practice make perfect? En J. A. Sloboda (Ed.), Generative Processes in Music: The psychology of performance, improvisation, and composition (pp. 91-112). Oxford: Clarendon Press.

Haddon, E. (2007). What does mental imagery mean to university music students and their professors? En A. Williamon y D. Coimbra (Eds.), Proceedings of the International Symposium on Performance Science 2007 (pp. 301-306). Utrecht, The Netherlands: European Association of Conservatoires (AEC).

Hallam, S. (1995). Professional musicians' orientations to practice: Implications for teaching. British Journal of Music Education, 12 (1), 3-20.

Hallam, S. (2001). The development of expertise in young musicians: Strategy use, knowledge acquisition and individual diversity. Music Education Research, 3 (1), 7-23.

Hallam, S. (2004). How important is practicing as a predictor of learning outcomes in instrumental music? En S. D. Lipscomb, R. Ashley, R. O. Gjerdingen y P. Webster (Eds.), Proceedings of the $8^{\text {th }}$ International Conference on Music Perception and Cognition (pp. 165168). Evanston, USA: Northwestern University.

Herrera, M. y Cremades, R. (2014). Memorisation in piano students: A study in the Mexican context. Musicae Scientiae, 2014, 18 (2), 216-231.

Hoppenot, D. (2000). El violín interior. Madrid: Real Musical.

Jørgensen, H. (2004). Strategies for individual practice. En A. Williamon (Ed.), Musical excellence. Strategies and techniques to enhance performance (pp. 85-103). London: Oxford University Press.

Jorquera, C. (2002). ¿Existe una didáctica del instrumento musical? Revista Electrónica de LEEME, 9. Recuperado de http://musica.rediris.es/leeme

THE CONTENT OF THIS ARTICLE IS THE SOLE RESPONSIBILITY OF THE AUTHORS. THE REVISTA ELECTRÓNICA DE LEEME AND UNIVERSITAT DE VALĖNCIA ARE NOT LIABLE FOR ANY LEGAL ACTIONS THAT MAY ARISE INVOLVING THE ARTICLE'S CONTENT. REVISTA ELECTRÓNICA DE LEEME -LISTA ELECTRÓNICA EUROPEA DE MÚSICA EN LA EDUCACIÓNHTTP://MUSICA.REDIRIS.ES.ISSN: 1575-9563 EDITORES: UNIVERSIDAD DE VALENCIA Y JESÚS TEJADA GIMÉNEZ. VISIBILIDAD DE ESTA REVISTA: EBSCO, CINDOC (CESIC), CITEFACTOR, COPAC, DIALNET, DICE (CSIC), DOAJ, E-REVISTAS (CSIC), EBSCO PREMIER, ERIH+, GALE CENGAGE LEARNING, IN-RECS, IRESIE, LATINDEX, MIAR, OCLC WORLDCAT, RESH, REDIB, RILM CORE JOURNALS, SUDOC, ULRICHS, ZEITSCHRIFTDATENBANK, ESTA REVISTA ESTÁ PUBLICADA CON EL APOYO INSTITUCIONAL DE REDIRIS-CONSEJO SUPERIOR DE INVESTIGACIONES CIENTIFICAS Y ES DE ACCESO LIBRE. 
Juslin, P. N. (2003). Five facets of musical expression: A psychologist's perspective on music performance. Psychology of Music, 31 (3), 273-302.

Juslin, P. N., Friberg, A., Schoonderwaldt, E. y Karlsson, J. (2004). Feedback learning of musical expressivity. En Williamon, A. (Ed.), Musical excellence. Strategies and techniques to enhance performance (pp. 247- 270). London: Oxford University Press.

Kenny, D., \& Ackermann, B. (2015). Performance-related musculoskeletal pain, depression and music performance anxiety in professional orchestral musicians: a population study. Psychology of Music, 43 (1), 43-60.

Klees-Dacheneder, U. y Campo A. C. (2000). Conseils pour l'entraînement mental quotidien. Revue Musicale Suisse, 1, 18.

Klöppel, R. (2005). Ejercitación mental para músicos. Barcelona: Ideabooks.

Kostka, M. J. (2002). Practice expectations and attitudes: a survey of college-level music teachers and students. Journal of Research in Music Education, 50 (2), 145-154.

Kurtz, G. (2007). Practicing: A Musician's Return to Music. New York: Borzoi Books.

Harnischmacher, C. (1997). The effects of individual differences in motivation, volition, and maturational processes on practice behavior of young instrumentalists. En H. Jørgensen y A. C. Lehmann (Eds.), Does practice make perfect? Current theory and research on instrumental music practice (pp. 71- 88). Norges: Norges musikkhøgskole.

Hinsom, M. (1995). Does your practice make perfect? Australian Music Teacher, 4 (4), 268.

Liertz, C. (2002). Developing performance confidence: A holistic training strategies program for managing practice and performance in music. Canberra, Australia: University of Canberra.

Lisboa, T., Chaffin, R., Schiaroli, A. G. y Barrera, A. (2004). Investigating practice and performance on the cello. En S. D. Lipscomb, R. Ashley, R. O. Gjerdingen y P. Webster (Eds.), Proceedings of the $8^{\text {th }}$ International Conference on Music Perception and Cognition (pp. 161164). Evanston, USA: Northwestern University.

Llorente, J. A. (2009). Julia Fischer: Futuro presente. Scherzo, 238, 52-55.

Macmillan, J. (2005). Strategies for memorising. ISM Music Journal, 1, 268-272.

MacRitchie, J., Buck, B. y Bailey, N. (2009). Visualising musical structure through performance gesture. En Proceedings of the 10th International Society for Music Information Retrieval Conference (pp141-163). Kobe, Japan: ISMIR.

Manresa, M. A. (2006). La actuación musical. Manual básico para interpretar en público. Barcelona: Editorial Boileau.

THE CONTENT OF THIS ARTICLE IS THE SOLE RESPONSIBILITY OF THE AUTHORS. THE REVISTA ELECTRÓNICA DE LEEME AND UNIVERSITAT DE VALĖNCIA ARE NOT LIABLE FOR ANY LEGAL ACTIONS THAT MAY ARISE INVOLVING THE ARTICLE'S CONTENT. REVISTA ELECTRÓNICA DE LEEME -LISTA ELECTRÓNICA EUROPEA DE MÚSICA EN LA EDUCACIÓN.HTTP://MUSICA.REDIRIS.ES.ISSN: 1575-9563 EDITORES: UNIVERSIDAD DE VALENCIA Y JESÚS TEJADA GIMÉNEZ. VISIBILIDAD DE ESTA REVISTA: EBSCO, CINDOC (CESIC), CITEFACTOR, COPAC, DIALNET, DICE (CSIC), DOAJ, E-REVISTAS (CSIC), EBSCO PREMIER, ERIH+, GALE CENGAGE LEARNING, IN-RECS, IRESIE, LATINDEX, MIAR, OCLC WORLDCAT, RESH, REDIB, RILM CORE JOURNALS, SUDOC, ULRICHS, ZEITSCHRIFTDATENBANK, ESTA REVISTA ESTA PUBLICADA CON EL APOYO INSTITUCIONAL DE REDIRIS-CONSEJO SUPERIOR DE INVESTIGACIONES CIENTIFICAS Y ES DE ACCESO LIBRE. 
Martenot, M. (1993). Principios fundamentales de formación musical y su aplicación. Madrid: Ediciones Rialp.

Mateo, A. (1997). Patrick Cohen, un músico sin prisa. Scherzo, 119, 135-138.

Mawer, D. (1999). Bridging the divide: embedding voice-leading analysis in string pedagogy and performance. British Journal of Music Education, 16 (2), 179- 195.

Mechner, F. (1995). Learning and practicing skilled performance. New York: The Mechner Foundation.

Michalak, J. (2006). Brass Basics Manual. Trumpet. Calgary: Foothills Brass Quintet - Brass Basics - JKM.

Miklaszewski, K. (1989). A case study of a pianist preparing a musical performance. Psychology of music, 17 (2), 95-109.

Miller, G. A. (1956). The magical number seven, plus or minus two: some limits on our capacity for processing information. Psychological Review, 63, 81-97.

Mishra, J. (2005). A theoretical model of musical memorization. Psychomusicology: Music, Mind and Brain, 19 (1), 75-89.

Mitchell, H. F. y MacDonald, R. A. (2009). Linguistic limitations of describing sound: Is talking about music like dancing about architecture? En A. Williamon, S. Pretty y R. Buck (Eds.), Proceedings of the International Symposium on Performance Science 2009 (pp. 45-50). Utrecht, The Netherlands: European Association of Conservatoires (AEC).

Narejos, A. (1998). Teoría y práctica de la ejecución pianística. Revista electrónica de LEEME, 1. Recuperado de http://musica.rediris.es/leeme

Narejos, A. (2000). Nueva mirada sobre la actividad del pianista. Revista electrónica de LEEME, 5. Recuperado de http://musica.rediris.es/leeme

Neuhaus, H. (1987). El arte del piano. Madrid: Real Musical.

Nieto, A. (1999). Contenidos de la técnica pianistica. Didáctica y aprendizaje. Barcelona: Editorial Boileau.

Nuki, M. (1984). Memorization of piano music. Psychologia: An International Journal of Psychology in the Orient, 27 (3), 157-163.

Ohgushi, K. (2006). Interaction between auditory and visual information in conveyance of players' intentions. Acoustical science and technology, 27 (6), 336-339.

THE CONTENT OF THIS ARTICLE IS THE SOLE RESPONSIBILITY OF THE AUTHORS. THE REVISTA ELECTRÓNICA DE LEEME AND UNIVERSITAT DE VALĖNCIA ARE NOT LIABLE FOR ANY LEGAL ACTIONS THAT MAY ARISE INVOLVING THE ARTICLE'S CONTENT. REVISTA ELECTRÓNICA DE LEEME -LISTA ELECTRÓNICA EUROPEA DE MÚSICA EN LA EDUCACIÓNHTTP://MUSICA.REDIRIS.ES.ISSN: 1575-9563 EDITORES: UNIVERSIDAD DE VALENCIA Y JESÚS TEJADA GIMÉNEZ. VISIBILIDAD DE ESTA REVISTA: EBSCO, CINDOC (CESIC), CITEFACTOR, COPAC, DIALNET, DICE (CSIC), DOAJ, E-REVISTAS (CSIC), EBSCO PREMIER, ERIH+, GALE CENGAGE LEARNING, IN-RECS, IRESIE, LATINDEX, MIAR, OCLC WORLDCAT, RESH, REDIB, RILM CORE JOURNALS, SUDOC, ULRICHS, ZEITSCHRIFTDATENBANK, ESTA REVISTA ESTÁ PUBLICADA CON EL APOYO INSTITUCIONAL DE REDIRIS-CONSEJO SUPERIOR DE INVESTIGACIONES CIENTIFICAS Y ES DE ACCESO LIBRE. 
Oyan, S. (2006). Mindfulness Meditation: Creative Musical Performance Through Awareness (Doctoral dissertation). Virginia: George Mason University.

Pachmann, V. (1913). Seeking originality. En J. F. Cooke (Ed.), Great Pianists on Piano Playing (pp. 182-196). Philadelphia: Theo. Presser.

Palmer, C. (1989). Mapping musical thought to musical performance. Journal of Experimental Psychology: Human Perception and Performance, 15 (2), 331-346.

Palmer, C. (1997). Music performance. Annual Review of Psychology, 48, 115-138.

Palmer, C., Carter, C., Koopmans, E. y Loehr, J. D. (2007). Movement, planning, and music: Motion coordinates of skilled performance. En Proceedings of the International Conference on Music Communication Science. Recuperado de http://marcs.uws.edu.au/links/ICoMusic/Full_Paper_PDF/Palmer_Carter_Koopmans_Loehr.pdf

Palmer, C. y Drake, C. (1997). Monitoring and planning capacities in the acquisition of music performance skills. Canadian Journal of Experimental Psychology, 51 (4), 369-384.

Palmer, C. y Hutchins, S. (2006). What is musical prosody? Psychology of learning and motivation, 46, 245-278.

Palmer, C., Koopmans, E., Carter, C., Loehr, J. D. y Wanderley, M. (2009). Synchronization of motion and timing in clarinet performance. En A. Williamon, S. Pretty y R. Buck (Eds.), Proceedings of the International Symposium on Performance Science 2009 (pp. 159-164). Utrecht, The Netherlands: European Association of Conservatoires (AEC).

Papageorgiou, G. (2007). Analyzing performance interpretation: The bouncing ball. En A. Williamon y D. Coimbra (Eds.), Proceedings of the International Symposium on Performance Science 2007 (pp.103-108). Utrecht, The Netherlands: European Association of Conservatoires (AEC).

Parncutt, R. (2003). Accents and expression in piano performance. En K. W. Niemöller (Ed.), Perspektiven und Methoden einer Systemischen Musikwissenschaft (pp. 163-185). Frankfurt: Peter Lang.

Parncutt, R. y Troup, M. (2002). Piano. En R. Parncutt y G. E. McPherson (Eds.), The Science and Psychology of Music Performance: Creative Strategies for Teaching and Learning (pp. 285-302). Oxford: Oxford University Press.

Pascual-Leone, A. (2003). The brain that makes music and is changed by it. En I. Peretz y R. J. Zatorre (Eds.), The Cognitive Neuroscience of Music (pp. 396-412). Oxford: Oxford University Press.

THE CONTENT OF THIS ARTICLE IS THE SOLE RESPONSIBILITY OF THE AUTHORS. THE REVISTA ELECTRÓNICA DE LEEME AND UNIVERSITAT DE VALĖNCIA ARE NOT LIABLE FOR ANY LEGAL ACTIONS THAT MAY ARISE INVOLVING THE ARTICLE'S CONTENT. REVISTA ELECTRÓNICA DE LEEME -LISTA ELECTRÓNICA EUROPEA DE MÚSICA EN LA EDUCACIÓN.HTTP://MUSICA.REDIRIS.ES.ISSN: 1575-9563 EDITORES: UNIVERSIDAD DE VALENCIA Y JESÚS TEJADA GIMÉNEZ. VISIBILIDAD DE ESTA REVISTA: EBSCO, CINDOC (CESIC), CITEFACTOR, COPAC, DIALNET, DICE (CSIC), DOAJ, E-REVISTAS (CSIC), EBSCO PREMIER, ERIH+, GALE CENGAGE LEARNING, IN-RECS, IRESIE, LATINDEX, MIAR, OCLC WORLDCAT, RESH, REDIB, RILM CORE JOURNALS, SUDOC, ULRICHS, ZEITSCHRIFTDATENBANK, ESTA REVISTA ESTÁ PUBLICADA CON EL APOYO INSTITUCIONAL DE REDIRIS-CONSEJO SUPERIOR DE INVESTIGACIONES CIENTIFICAS Y ES DE ACCESO LIBRE. 
Pascuali, G. y Príncipe, R. (1982). El violín. Manual de cultura y práctica violinística. Buenos Aires: Editorial Ricordi.

Pérez Gómez, A. (1983). Paradigmas contemporáneos de investigación didáctica. En J. Gimeno y A. Pérez (Eds.), La enseñanza: su teoría y su práctica (pp. 95- 138). Madrid: Akal.

Pérez Sánchez, M. (2003). El piano con corazón. Quodlibet: Revista de especialización musical, 27, 71-97.

Pertzborn, F., Coimbra, D., Hallam, S. y Braga, A. C. (2009). Developing the ability to perform: Investigating the field of higher education and expertise development for learning and performing the double bass. En A. Williamon, S. Pretty y R. Buck (Eds.), Proceedings of the International Symposium on Performance Science 2009 (pp. 133-138). Utrecht, The Netherlands: European Association of Conservatoires (AEC).

Pfordresher, P. Q. (2006). Coordination of perception and action in music performance. Advances in Cognitive Psychology, 2 (2-3), 183-198.

Pin, A. (2001). Entrevista a Emilio Colón. Revista filomúsica, 22. Recuperado de http://www.filomusica.com

Pitts, S. E., Davidson, J. W. y McPherson, G. E. (2002). Developing effective practice strategies: Case studies of three young instrumentalists. En G. Spruce (Ed.), Aspects of teaching secondary music: perspectives on practice (pp. 140-152). London: RoutledgeFalmer.

Pozo, J. I. (1989). Teorías cognitivas del aprendizaje. Madrid: Ediciones Morata.

Pozo, J. I., Bautista, A. y Torrado, J. A. (2008). El aprendizaje y la enseñanza de la interpretación musical: cambiando las concepciones y las prácticas. Cultura y Educación, 20 (1), 5-15.

Pozo, J. I. y Monereo, C. (Coords.) (1999). El aprendizaje estratégico: enseñar a aprender desde el currículo. Madrid: Santillana.

Ramos, C. M. (1981). La dinámica del violinista. Buenos Aires: Ed. Ricordi.

Rauschecker, J. P. (2003). Functional organization and plasticity of auditory cortex. En I. Peretz y R. J. Zatorre (Eds.), The Cognitive Neuroscience of Music (pp. 357-365). Oxford: Oxford University Press.

Reid, S. (2006). Preparándose para interpretar. En J. Rink (Ed.), La interpretación musical (pp. 125-136). Madrid: Alianza Música.

Renwick, J. M. y McPherson, G.E. (2002). Interest and choice: Student-selected repertoire and its effect on practicing behavior. British Journal of Music Education, 19 (2), 173-188.

THE CONTENT OF THIS ARTICLE IS THE SOLE RESPONSIBILITY OF THE AUTHORS. THE REVISTA ELECTRÓNICA DE LEEME AND UNIVERSITAT DE VALĖNCIA ARE NOT LIABLE FOR ANY LEGAL ACTIONS THAT MAY ARISE INVOLVING THE ARTICLE'S CONTENT. REVISTA ELECTRÓNICA DE LEEME -LISTA ELECTRÓNICA EUROPEA DE MÚSICA EN LA EDUCACIÓNHTTP://MUSICA.REDIRIS.ES.ISSN: 1575-9563 EDITORES: UNIVERSIDAD DE VALENCIA Y JESÚS TEJADA GIMÉNEZ. VISIBILIDAD DE ESTA REVISTA: EBSCO, CINDOC (CESIC), CITEFACTOR, COPAC, DIALNET, DICE (CSIC), DOAJ, E-REVISTAS (CSIC), EBSCO PREMIER, ERIH+, GALE CENGAGE LEARNING, IN-RECS, IRESIE, LATINDEX, MIAR, OCLC WORLDCAT, RESH, REDIB, RILM CORE JOURNALS, SUDOC, ULRICHS, ZEITSCHRIFTDATENBANK, ESTA REVISTA ESTÁ PUBLICADA CON EL APOYO INSTITUCIONAL DE REDIRIS-CONSEJO SUPERIOR DE INVESTIGACIONES CIENTIFICAS Y ES DE ACCESO LIBRE. 
Repp, B. H. (1992). Diversity and commonality in music performance: An analysis of timing microstructure in Schumann's "Traumerei". Haskins Laboratories Status Report on Speech Research, SR-111/112, 227-260.

Repp, B. H. (2001). Expressive Timing in the Mind's Ear. En R. I. Godøy y H. Jørgensen (Eds.), Musical Imagery (pp. 185-200). Lisse, The Netherlands: Swets and Zeitlinger.

Romero, J. (1994). András Schiff: el hombre tranquilo. Revista Scherzo, 90, 39-46.

Rosenbaum, D. A., Kenny, S. B. y Derr, M. A. (1983). Hierarchical control of rapid movement sequences. Journal of Experimental Psychology: Human Perception and Performance, 9 (1), 86-102.

Ross, S. L. (1985). The Effectiveness of Mental Practice in Improving the Performance of College Trombonists. Journal of Research in Music Education, 33, 223-229.

Rosset-Llobet, J. (2000). Identification of Risk Factors for Musicians in Catalonia (Spain). Medical Problems of Performing Artists, 15, 167-174.

Rubin-Rabson, G. (1941). Studies in the psychology of memorizing piano music. V: A comparison of pre-study periods of varied length. Journal of Educational Psychology, 32, 101112.

Ruiz, G. (1999). Amo hacer música. Yogaterapia especifica para los profesionales de la música. Madrid: Mandala Ediciones.

Ruiz, M. H., Jabusch, H. C. y Altenmüller, E. (2009). Fast feedforward error-detection mechanisms in highly skilled music performance. En A. Williamon, S. Pretty y R. Buck (Eds.), Proceedings of the International Symposium on Performance Science 2009 (pp. 187-197). Utrecht, The Netherlands: European Association of Conservatoires (AEC).

Salazar, J. F. y Contreras, L. S. (2007). Miedo escénico en músicos académicos de Caracas. Caracas: Universidad Central de Venezuela. Facultad de Humanidades y Educación.

Shaffer, L. H. (1980). Analysing piano performance: a study of concert pianists. Advances in Psychology: Tutorials in Motor Behavior, 1, 443-455.

Samama-Polak, A. L. (1990). El stress y los músicos. Algunos consejos válidos para todos los intérpretes. Música y Educación: Revista internacional de pedagogía musical, 3 (2), 299-312.

Sánchez, C. A. (2006). Un análisis de los aspectos comunicativos del pianista Vladimir Horowitz con el público. Revista Electrónica de LEEME, 17. Recuperado de http://musica.rediris.es/leeme

THE CONTENT OF THIS ARTICLE IS THE SOLE RESPONSIBILITY OF THE AUTHORS. THE REVISTA ELECTRÓNICA DE LEEME AND UNIVERSITAT DE VALĖNCIA ARE NOT LIABLE FOR ANY LEGAL ACTIONS THAT MAY ARISE INVOLVING THE ARTICLE'S CONTENT. REVISTA ELECTRÓNICA DE LEEME -LISTA ELECTRÓNICA EUROPEA DE MÚSICA EN LA EDUCACIÓN.HTTP://MUSICA.REDIRIS.ES.ISSN: 1575-9563 EDITORES: UNIVERSIDAD DE VALENCIA Y JESÚS TEJADA GIMÉNEZ. VISIBILIDAD DE ESTA REVISTA: EBSCO, CINDOC (CESIC), CITEFACTOR, COPAC, DIALNET, DICE (CSIC), DOAJ, E-REVISTAS (CSIC), EBSCO PREMIER, ERIH+, GALE CENGAGE LEARNING, IN-RECS, IRESIE, LATINDEX, MIAR, OCLC WORLDCAT, RESH, REDIB, RILM CORE JOURNALS, SUDOC, ULRICHS, ZEITSCHRIFTDATENBANK, ESTA REVISTA ESTÁ PUBLICADA CON EL APOYO INSTITUCIONAL DE REDIRIS-CONSEJO SUPERIOR DE INVESTIGACIONES CIENTIFICAS Y ES DE ACCESO LIBRE. 
Sánchez Huete, J. C. (2008). Conocimiento científico de la didáctica. En Sánchez Huete, J. C. (Coord.), Compendio de didáctica general (pp. 49-72). Madrid: Editorial CCS.

Sauer, E. (1913). The training of the virtuoso. En J. F. Cooke (Ed.), Great Pianists on Piano Playing (pp. 236-251). Philadelphia: Theo. Presser.

Schelling, E. (1913). Learning a new piece. En J. F. Cooke (Ed.), Great Pianists on Piano Playing (pp. 267-278). Philadelphia: Theo. Presser.

Sloboda, J. A. (1983). The communication of musical metre in piano performance. The Quarterly Journal of Experimental Psychology, 35 (2), 377-396.

Sloboda, J. A. (1991). Music Structure and Emotional Response: Some Empirical Findings. Psychology of Music, 19 (2), 110-120.

Sloboda, J. A., Davidson, J. W., Howe, M. J. A. y Moore, D. G. (1996). The role of practice in the development of performing musicians. British Journal of Psychology, 87, 287-309.

Sloboda, J. A., Minassian, C. y Gayford, C. (2003). Assisting advanced musicians to enhance their expressivity - An intervention study. En R. Kopiez, A. C. Lehmann, I. Wolther y C. Wolf (Eds.), Proceedings of the Fifth Triennial ESCOM Conference (p. 92). Hanover, Germany: Hanover University of Music and Drama.

Steiner, R. (1984). Cómo se adquiere el conocimiento de los mundos superiores. Buenos Aires: Dedalo.

Stoeber, J. y Eismann, U. (2007). Perfectionism in young musicians: Relations with motivation, effort, achievement, and distress. Personality and Individual Differences, 43 (8), 2182- 2192.

Stravinsky, I. (1977). Poética musical. Madrid: Taurus.

Todd, N. P. M. (1995). The kinematics of musical expression. Journal of the Acoustical Society of America, 97, 1940-1949.

Torrado, J. A., Casas, A. y Pozo, J. I. (2005). Las culturas de la educación musical: aprendiendo a interpretar un instrumento. Estudios de Psicología, 26 (2), 259-269.

Tripiana, S. (2012). Estrategias eficaces de práctica instrumental. Revista Música y Educación, 91, 64-72.

Tripiana, S. (2016). Importancia de las estrategias de práctica instrumental en la educación musical superior. Revista Electrónica Complutense de Investigación en Educación MusicalRECIEM, 13, 64-88.

Trusheim, W. H. (1991). Audiation and mental imagery: Implications for artistic performance. The Quarterly, 2 (1-2), 138-147.

THE CONTENT OF THIS ARTICLE IS THE SOLE RESPONSIBILITY OF THE AUTHORS. THE REVISTA ELECTRÓNICA DE LEEME AND UNIVERSITAT DE VALĖNCIA ARE NOT LIABLE FOR ANY LEGAL ACTIONS THAT MAY ARISE INVOLVING THE ARTICLE'S CONTENT. REVISTA ELECTRÓNICA DE LEEME -LISTA ELECTRÓNICA EUROPEA DE MÚSICA EN LA EDUCACIÓN.HTTP://MUSICA.REDIRIS.ES.ISSN: 1575-9563 EDITORES: UNIVERSIDAD DE VALENCIA Y JESÚS TEJADA GIMÉNEZ. VISIBILIDAD DE ESTA REVISTA: EBSCO, CINDOC (CESIC), CITEFACTOR, COPAC, DIALNET, DICE (CSIC), DOAJ, E-REVISTAS (CSIC), EBSCO PREMIER, ERIH+, GALE CENGAGE LEARNING, IN-RECS, IRESIE, LATINDEX, MIAR, OCLC WORLDCAT, RESH, REDIB, RILM CORE JOURNALS, SUDOC, ULRICHS, ZEITSCHRIFTDATENBANK, ESTA REVISTA ESTÁ PUBLICADA CON EL APOYO INSTITUCIONAL DE REDIRIS-CONSEJO SUPERIOR DE INVESTIGACIONES CIENTIFICAS Y ES DE ACCESO LIBRE. 
Vigotsky, L. S. (1986). Aprendizaje y desarrollo intelectual en la edad escolar. En A. R. Luria, A. N. Leontiev, L. S. Vigotsky et al. (Comp.), Psicología y pedagogía (pp. 438-452). Madrid: Akal.

Vila, T. (2007). Assessing the importance of visual/theatrical features in the perception of music by an audience, using sociological tools. En A. Williamon y D. Coimbra (Eds.), Proceedings of the International Symposium on Performance Science 2007 (pp.147-152). Utrecht, The Netherlands: European Association of Conservatoires (AEC).

Vines, B. W., Krumhansl, C. L., Wanderley, M. M. y Levitin, D. J. (2006). Cross-modal interactions in the perception of musical performance. Cognition, 101, 80-113.

Wanderley, M. M. (2002). Quantitative analysis of non-obvious performer gestures. En I. Wachsmuth y T. Sowa (Eds.), Gesture and Sign Language in Human-Computer Interaction (pp. 241-253). Berlin, Heidelberg: Springer Verlag.

Weaver, J. L. (2005). The role of goals and practice steps in piano practice assignments. Ohio: Bowling Green State University.

Willems, E. (1994). El valor humano de la educación musical. Barcelona: Ediciones Paidós Ibérica.

Williamon, A. (Ed.). (2004). Musical Excellence. Strategies and techniques to enhance performance. London: Oxford University Press.

Williamon, A. y Valentine, E. (2000). Quantity and quality of musical practice as predictors of performance quality. British Journal of Psychology, 91, 353-376.

Williamon, A. y Valentine, E. (2002). The role of retrieval structures in memorizing music. Cognitive Psychology, 44, 1-32.

Wilson, G. D. y Roland, D. (2002). Performance anxiety. En R. Parncutt y G. E. McPherson (Eds.), The Science and Psychology of Music Performance: Creative Strategies for Teaching and Learning (pp. 47-61). Oxford: Oxford University Press.

Woody, R. H. (2004). Advanced music students' use of imagery and metaphor-based instruction in generating expressive performance. En S. D. Lipscomb, R. Ashley, R. O. Gjerdingen y P. Webster (Eds.), Proceedings of the $8^{\text {th }}$ International Conference on Music Perception and Cognition (pp. 482-485). Adelaide, Australia: Causal Productions.

Wrigley, W. J. y Emmerson, S. B. (2013). The experience of the flow state in live music performance. Psychology of Music, 41 (3), 292-305.

Wulf, G. y Mornell, A. (2008). Insights about practice from the perspective of motor learning: a review. Music Performance Research, 2, 1-25

THE CONTENT OF THIS ARTICLE IS THE SOLE RESPONSIBILITY OF THE AUTHORS. THE REVISTA ELECTRÓNICA DE LEEME AND UNIVERSITAT DE VALĖNCIA ARE NOT LIABLE FOR ANY LEGAL ACTIONS THAT MAY ARISE INVOLVING THE ARTICLE'S CONTENT. REVISTA ELECTRÓNICA DE LEEME -LISTA ELECTRÓNICA EUROPEA DE MÚSICA EN LA EDUCACIÓN.HTTP://MUSICA.REDIRIS.ES.ISSN: 1575-9563 EDITORES: UNIVERSIDAD DE VALENCIA Y JESÚS TEJADA GIMÉNEZ. VISIBILIDAD DE ESTA REVISTA: EBSCO, CINDOC (CESIC), CITEFACTOR, COPAC, DIALNET, DICE (CSIC), DOAJ, E-REVISTAS (CSIC), EBSCO PREMIER, ERIH+, GALE CENGAGE LEARNING, IN-RECS, IRESIE, LATINDEX, MIAR, OCLC WORLDCAT, RESH, REDIB, RILM CORE JOURNALS, SUDOC, ULRICHS, ZEITSCHRIFTDATENBANK, ESTA REVISTA ESTÁ PUBLICADA CON EL APOYO INSTITUCIONAL DE REDIRIS-CONSEJO SUPERIOR DE INVESTIGACIONES CIENTIFICAS Y ES DE ACCESO LIBRE. 
LISTA ELECTRÓNICA EUROPEA

DE MÚSICA EN LA EDUCACIÓN

Zarza-Alzugaray, F. J., Casanova-López, O., y Robles-Rubio, J. E. (2016). Relación entre ansiedad escénica, perfeccionismo y calificaciones en estudiantes del Titulo Superior de Música. ReiDoCrea, 5, 16-21.

Zatorre, R. J. y Halpern, A. R. (2005). Mental concerts: musical imagery and auditory cortex. Neuron, 47 (1), 9-12. 